\title{
PRE-ENGINEERING SPACEFLIGHT VALIDATION OF ENVIRONMENTAL MODELS AND THE 2005 HZETRN SIMULATION CODE
}

\author{
J.E. Nealy ${ }^{\mathrm{a}}$, F.A. Cucinotta ${ }^{\mathrm{b}}$, J.W. Wilson ${ }^{\mathrm{c}}$, F.F. Badavi ${ }^{\mathrm{d}}$,Ts.P. Dachev ${ }^{\mathrm{e}}$, B.T. Tomov ${ }^{\mathrm{e}}$, S.A. Walker ${ }^{\mathrm{a}}$, \\ G. De Angelis ${ }^{\mathrm{a}, \mathrm{f}}$, S.R. Blattnig ${ }^{\mathrm{c}}$, W. Atwell ${ }^{\mathrm{g}}$ \\ ${ }^{a}$ Old Dominion University, Norfolk, VA USA \\ ${ }^{\mathrm{b}}$ NASA Johnson Space Center, Houston, TX USA \\ ${ }^{\mathrm{c}}$ NASA Langley Research Center, Hampton, VA USA \\ ${ }^{\mathrm{d}}$ Christopher Newport University, Newport News, VA USA \\ ${ }^{\mathrm{e}}$ Bulgarian Academy of Science, Sofia, BG \\ ${ }^{\mathrm{f}}$ Istituto Superiore di Sanità, Rome, Italy \\ ${ }^{\mathrm{g}}$ The Boeing Company, Houston, TX USA \\ john.w.wilson@nasa.gov/ Fax: +1-757-864-8094
}

Abstract

The HZETRN code has been identified by NASA for engineering design in the next phase of space exploration highlighting a return to the Moon in preparation for a Mars mission. In response, a new series of algorithms beginning with 2005 HZETRN, will be issued by correcting some prior limitations and improving control of propagated errors along with established code verification processes. Code validation processes will use new/improved low Earth orbit (LEO) environmental models with a recently improved International Space Station (ISS) shield model to validate computational models and procedures using measured data aboard ISS. These validated models will provide a basis for flight-testing the designs of future space vehicles and systems of the Constellation program in the LEO environment.

\section{Introduction}

Improved spacecraft shield design requires early entry of radiation constraints into the design process to maximize performance and minimize costs. As a result, we have been investigating computational procedures to allow shield analysis starting with preliminary design concepts through high-fidelity final design models (Wilson et al. 2003). Of particular importance is the need to implement probabilistic models to account for design uncertainties (Wilson et al. 2004) in the context of optimal design processes (Qualls et al. 2003). These requirements need supporting tools with high computational efficiency to enable appropriate design methods. Only the HZETRN code has so far been identified for this purpose. As a result, Wilson et al. (2005) have prepared a review of past HZETRN code development, verification, and validation. Although there has been sporadic research to generalize this code over the last ten years, evaluation of code status among principal users demonstrated drift in the various code versions and databases. As a result of this renewed interest in HZETRN for future space systems design, there is now a systematic effort to advance, verify, and validate these codes in preparation to integrating them into engineering design processes. The present paper will describe the first several months of this renewed systematic effort.

As NASA's newly defined technology development spirals are now progressing, there is a need to identify a suitable code for the early spiral processes (development of a Crew Exploration Vehicle, CEV). We have chosen 
the 1995 HZETRN version for this early spiral development. This code has had wide distribution and is largely the code used in the prior verification and validation processes described by Wilson et al. (2005). However, an assessment of the 1995 HZETRN code status demands the re-evaluation of the computational procedures and databases to account for software and database drift over the last ten years and the inherent limitations of these prior computational procedures. In the present paper, we review the basic derivations to identify oversights and improve solution stability and accuracy. In prior papers, we used both analytical and Monte Carlo benchmarks to both identify problems among various versions. We will distribute these benchmarks with the code package to evaluate future compiler and platform dependent problems in addition to future drift for whatever reason. This revised 1995 HZETRN code, chronicled as the 2005 HZETRN (Wilson et al. 2006a), is recommended for standard practice and the present paper will focus on using measurements aboard ISS and recently improved models of the low Earth orbit (LEO) environment (Wilson et al. 2006b) for code validation. In addition to adding directional dependence and solar modulation to the improved trapped proton model AP8, we further developed a directional dependent geomagnetic transmission model. The main effect of this directional dependence of geomagnetic transmission is the penetration of particles below the vertical cutoff transmission model used in the past. Furthermore, we provide for the dynamic behavior of the Earth's magnetic field covering the period 1945 to 2020. The emphasis of the present paper is to validate the modulated environmental models and 2005 HZETRN shielding code using detailed measurements on ISS.

\section{2005 HZETRN}

The specification of the interior environment within a spacecraft and evaluation of the effects on the astronaut is at the heart of the space radiation protection problem. The Langley Research Center has been developing such techniques and an in-depth presentation is given in Wilson et al. (1991), although considerable progress has been made since that publication. The relevant transport equations are the coupled linear Boltzmann equations for a closed convex domain (Fig. 1) derived on the basis of conservation principles for the flux density (particles $/ \mathrm{cm}^{2}$-sr-s-A-MeV) $\phi_{j}(\boldsymbol{x}, \boldsymbol{O}, E$ ) for particle type $j$ as

$$
\boldsymbol{O} \bullet \boldsymbol{\nabla} \phi_{j}(\boldsymbol{x}, \boldsymbol{O}, E)=\sum_{k} \int \sigma_{j k}\left(\boldsymbol{O}, \boldsymbol{O}^{\prime}, E, E^{\prime}\right) \phi_{k}\left(\boldsymbol{x}, \boldsymbol{O}^{\prime}, E^{\prime}\right) d \boldsymbol{O}^{\prime} d E^{\prime}-\sigma_{j}(E) \phi_{j}(\boldsymbol{x}, \boldsymbol{O}, E)
$$

where $\sigma_{j}(E)$ and $\sigma_{j k}\left(\boldsymbol{O}, \boldsymbol{O}^{\prime}, E, E^{\prime}\right)$ are the shield media macroscopic cross sections. The $\sigma_{j k}\left(\boldsymbol{O}, \boldsymbol{O}^{\prime}, E, E^{\prime}\right)$ represent all those processes by which type $k$ particles moving in direction $\boldsymbol{\Omega}^{\prime}$ with energy $E^{\prime}$ produce a type $j$ particle in direction $\boldsymbol{O}$ with energy $E$ (including decay processes). Note that there may be several reactions that produce a particular product, and the appropriate cross sections for equation (1) are the inclusive ones. Exclusive processes are functions of the particle fields and may be included once the particle fields are known. Note, we will at times loosely refer to $\phi_{j}(\boldsymbol{x}, \boldsymbol{O}, E)$ as either flux or fluence and the usage should be clear from the context. The time scale of the processes in equation (1) are at most on the order of microseconds while time scales of boundary conditions are on the order of minutes or longer leaving the resulting interior fields in equilibrium with the particles at the bounding surface.

The total cross section $\sigma_{j}(E)$ with the medium for each particle type is

$$
\sigma_{j}(E)=\sigma_{j}^{a t}(E)+\sigma_{j}^{e l}(E)+\sigma_{j}^{r}(E)
$$

where the first term refers to collision with atomic electrons, the second term is for elastic scattering on the nucleus, and the third term describes nuclear reactions where we have ignored the minor nuclear inelastic processes (excited single particle states) except for low energy neutron collisions. The

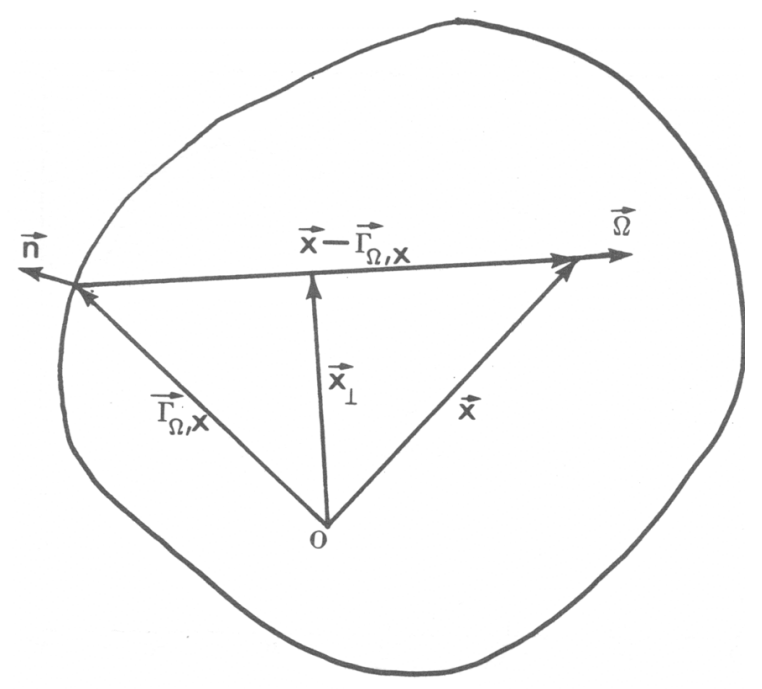

Fig. 1 Geometric relations of quantities useful in solving equation (1). corresponding differential cross sections are similarly ordered. Many atomic collisions $\left(\sim 10^{6}\right)$ occur in a centimeter of ordinary matter, whereas $\sim 10^{3}$ nuclear coulomb elastic collisions occur per centimeter, while nuclear scattering and reactive collisions are separated by a fraction to many centimeters depending on energy and particle type. We include in the $\sigma_{j}^{r}(E)$ term the nuclear decay processes. Solution methods first use physical perturbations based on the ordering of the cross sections with the frequent atomic interactions as the first physical perturbation with special methods used for neutrons for which atomic cross sections are zero. The first physical perturbation to be treated is 
the highly directed atomic collisions with mean free paths on the order of micrometers as observed in nuclear emulsion. The usual approximation is the continuous slowing down approximation leading to well specified rangeenergy relations but neglects the energy straggling that will be discussed later (see also Wilson et al. 2002a). The next term is the highly directed multiple coulomb scattering and is usually neglected in many models but is of great importance in understanding the transport of unidirectional ion beams leading to beam divergence and is treated in detail elsewhere (Shavers et al. 1993). The remaining nuclear reactive processes have been given main attention in past code developments. We will now consider the formal development of the relevant equations for further consideration.

\subsection{Continuous Slowing Down Approximation}

The collisions with atomic electrons preserve the identity of the ion and the differential cross sections are given as

$$
\sigma_{j k}^{a t}\left(\boldsymbol{O}, \boldsymbol{O}^{\prime}, E, E^{\prime}\right)=\Sigma_{n} \sigma_{j, n}^{a t}\left(E^{\prime}\right) \delta\left(\boldsymbol{O}-\boldsymbol{O}^{\prime}\right) \delta_{j k} \delta\left(E+A_{j}^{-1} \varepsilon_{n}-E^{\prime}\right)
$$

where $n$ refers to the atomic/molecular excited states with excitation energies $\varepsilon_{\mathrm{n}}$ including the continuum. Note, the factor $A_{j}^{-1}$ results from the units of $E$ of $\mathrm{A} \mathrm{MeV} \mathrm{(equivalent} \mathrm{unit} \mathrm{of} \mathrm{MeV} /$ nucleon). Although the atomic/molecular cross-sections $\sigma_{j, n}^{a t}\left(E^{\prime}\right)$ are large $\left(\approx 10^{-16} \mathrm{~cm}^{2}\right)$, the energy transfers $\varepsilon_{n}$ are small $(\approx 1-100 \mathrm{eV})$ compared to the particle energy. The atomic/molecular terms of equation (1) may be written as

$$
\begin{aligned}
\sum \int \sigma^{a t}{ }_{j k}\left(\boldsymbol{O}, \boldsymbol{O}^{\prime}, E, E^{\prime}\right) \phi_{k}\left(\boldsymbol{x}, \boldsymbol{O}^{\prime}, E^{\prime}\right) & d \boldsymbol{O}^{\prime} d E^{\prime}-\sigma^{a t}{ }_{j}(E) \phi_{j}(\boldsymbol{x}, \boldsymbol{Q}, E)=\Sigma_{n} \sigma^{a t}{ }_{j, n}\left(E+A_{j}^{-1} \varepsilon_{n}\right) \phi_{j}\left(\boldsymbol{x}, \boldsymbol{O}, E+A_{j}^{-1} \varepsilon_{n}\right)-\sigma^{a t}(E) \phi_{j}(\boldsymbol{x}, \boldsymbol{O}, E) \\
& =\Sigma_{n}\left\{\sigma^{a t}{ }_{j, n}(E) \phi_{j}(\boldsymbol{x}, \boldsymbol{O}, E)+A_{j}^{-1} \varepsilon_{n} \partial_{E}\left[\sigma^{a t}{ }_{j, n}(E) \phi_{j}(\boldsymbol{x}, \boldsymbol{O}, E)\right]\right\}-\sigma^{a t}{ }_{j}(E) \phi_{j}(\boldsymbol{x}, \boldsymbol{O}, E)+O\left(\varepsilon_{n}{ }^{2}\right) \\
& =\partial_{E}\left[S_{j}(E) \phi_{j}(\boldsymbol{x}, \boldsymbol{O}, E)\right]+O\left(\varepsilon_{n}{ }^{2}\right)
\end{aligned}
$$

where the stopping power $S_{j}(E)$ is given as the sum of energy transfers and atomic excitation cross sections as

$$
S_{j}(E)=\Sigma_{n} \varepsilon_{n} \sigma_{j, n}^{a t}(E)
$$

The higher order terms of equation (4) give rise to straggling and are neglected in the continuous slowing down approximation (csda) but are discussed elsewhere (Wilson et al. 2002a, Tweed et al. 2005). Evaluation of the stopping power by equation (5) is deceptively simple in that all of the excited states including continuum states of the atomic/molecular system need to be known. Furthermore, the projectile remains a bare ion except at low energies where the projectile ion atomic orbital states begin to resonate with the electrons of the media leading to electron capture and lowering of the ion charge. These issues are further discussed in Wilson et al. (1991) and Tai et al. (1997) in regard to the atomic data base. Equation (1) can be written in the csda as

$$
\boldsymbol{O} \bullet \boldsymbol{\nabla} \phi_{j}(\boldsymbol{x}, \boldsymbol{O}, E)-A_{j}^{-1} \partial_{E}\left[S_{j}(E) \phi_{j}(\boldsymbol{x}, \boldsymbol{O}, E)\right]=\sum \int \sigma_{j k}\left(\boldsymbol{O}, \boldsymbol{O}^{\prime}, E, E^{\prime}\right) \phi_{k}\left(\boldsymbol{x}, \boldsymbol{O}^{\prime}, E^{\prime}\right) d \boldsymbol{O}^{\prime} d E^{\prime}-\sigma_{j}(E) \phi_{j}(\boldsymbol{x}, \boldsymbol{O}, E)
$$

where the right-hand side of equation (6) excludes the atomic/molecular processes now appearing on the left as an energy shifting operator in addition to the usual spatial drift term. Neutral particles would have null atomic cross sections for which the stopping term of equation (6) does not appear. Application of csda in both laboratory and space shielding has been wide and the resulting errors are discussed elsewhere (Tweed et al. 2005). Equation (6) can be rewritten as an integral equation (Wilson 1977) as

$$
\begin{aligned}
\phi_{j}(\boldsymbol{x}, \boldsymbol{Q}, E) & =\left\{S_{j}\left(E_{\nu}\right) P_{j}\left(E_{\gamma}\right) \phi_{j}\left(\boldsymbol{\Gamma}(\boldsymbol{Q}, \boldsymbol{x}), \boldsymbol{Q}, E_{\nu}\right)\right. \\
& +\sum \int_{E}^{E_{\gamma}} d E^{\prime} A_{j} P_{j}\left(E^{\prime}\right) \int_{E^{\prime}}{ }^{\infty}{ }_{4 \pi} d E^{\prime \prime} d \boldsymbol{Q}^{\prime} \sigma_{j k}\left(\boldsymbol{Q}, \boldsymbol{O}^{\prime}, E^{\prime}, E^{\prime \prime}\right) \\
& \left.\boldsymbol{x} \phi_{k}\left(\boldsymbol{x}+\left[R_{j}(E)-R_{j}\left(E^{\prime}\right)\right] \boldsymbol{Q}, \boldsymbol{Q}^{\prime}, E^{\prime \prime}\right)\right\} / S_{j}(E) P_{j}(E)
\end{aligned}
$$

where $\boldsymbol{\Gamma}$ is the point on the boundary connected to $\boldsymbol{x}$ along direction - $\mathbf{Q}, E_{\gamma}=R_{j}^{-1}\left[\rho-d+R_{j}\right], \rho$ is the projection of $\mathbf{x}$ onto $\mathbf{Q}$ (Fig. 1), $d$ is the projection of $\boldsymbol{\Gamma}$ onto $\boldsymbol{Q}, R_{j}(E)$ is the distance an ion of type $j$ of energy $E$ will travel before losing all of its energy to excitation of atomic electrons, and $P_{j}(E)$ is the probability a type $j$ ion of energy $E$ will have a nuclear reaction in coming to rest in the media. The usual rangeenergy relation is given by

$$
R_{j}(E)=\int A_{j} d E^{\prime} / S_{j}\left(E^{\prime}\right)
$$

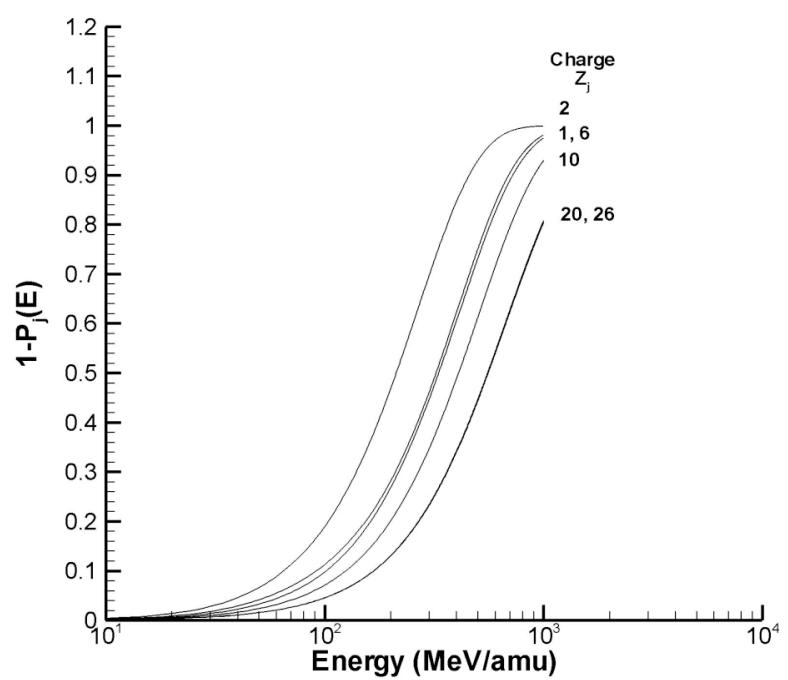

Fig. 2 Probability of nuclear reaction as a function of ion type and energy. 
The nuclear attenuation function (Fig. 2) is given by

$$
P_{j}(E)=\exp \left[-\int A_{j} \sigma_{j}\left(E^{\prime}\right) d E^{\prime} / S_{j}\left(E^{\prime}\right)\right]
$$

where the integral domains in equations (8) and (9) extend over the full energy range $\{0, E\}$.

\subsection{Straightahead Approximation}

The approach to a practical solution of equation (7) is to develop a progression of solutions from the simple to the complex allowing early implementation of high-performance computational procedures and establishing a converging sequence of approximations with established accuracy criteria and means of verification. The lowest order approximation using the straightahead approximation was guided by the nucleon transport studies of Alsmiller et al. (1968) using the Monte Carlo methods in which the differential cross sections were approximated as

$$
\sigma_{j k}^{r}\left(\boldsymbol{Q}, \boldsymbol{Q}^{\prime}, E, E^{\prime}\right) \approx \sigma_{j k}^{r}\left(E, E^{\prime}\right) \delta\left(\boldsymbol{O}-\boldsymbol{Q}^{\prime}\right)
$$

resulting in dose and dose equivalent per unit fluence to be within the statistical uncertainty of the Monte Carlo result obtained using the fully angle dependent cross sections. The relation of angular dependent cross sections to spacecraft geometry in space application was further examined by Wilson and Kandelwal (1974) using asymptotic expansions about angular divergence parameters demonstrating errors in the straightahead approximation to be on the order of the square of the ratio of distance of divergence to radius of curvature of the shield (a small error in most space systems).

Equations (6) and (7) were examined for HZE ions using the following form for the projectile fragmentation cross sections as

$$
\sigma_{j k}^{r}\left(\boldsymbol{O}, \boldsymbol{O}^{\prime}, E, E^{\prime}\right)=\sigma_{j k}^{r}\left(E^{\prime}\right) N_{t} \exp \left\{-\left[\boldsymbol{O} \sqrt{E}-\boldsymbol{O}^{\prime} \sqrt{\left.E^{\prime}\right]^{2} / 2} \varepsilon_{j k}^{2}\right\}\right.
$$

where $\sigma_{j k}^{r}\left(E^{\prime}\right)$ is the cross section for producing fragment $j$ from ion $k, N_{t}$ is the normalization constant for the exponential function, and $\varepsilon_{j k}$ is the momentum dispersion parameter in the reaction. Substituting the interactive form of equation (11) into the integral term (target fragments treated separately, Wilson 1977) of the Boltzmann equation (6) yields

$$
\begin{aligned}
\sum \int \sigma_{j k}^{r}\left(\boldsymbol{O}, \boldsymbol{O}^{\prime}, E, E^{\prime}\right) \phi_{k}\left(\boldsymbol{x}, \boldsymbol{O}^{\prime}, E^{\prime}\right) d \boldsymbol{O}^{\prime} d E^{\prime}= & \sum \sigma_{j k}^{r}\left(E^{\prime}\right)\left\{\phi_{k}(\boldsymbol{x}, \boldsymbol{O}, E)+E \partial_{E} \phi_{k}(\boldsymbol{x}, \boldsymbol{O}, E) \sqrt{ }\left[\varepsilon_{j k}^{2} /(2 m E)\right]\right. \\
& \left.+\boldsymbol{\boldsymbol { O } \bullet \boldsymbol { \partial } _ { \boldsymbol { O } }} \phi_{k}(\boldsymbol{x}, \boldsymbol{O}, E) \varepsilon_{j k}^{2} /(2 m E)\right\}
\end{aligned}
$$

where the second term on the right hand side of equation (12) results from corrections in assuming the velocity of the ion is preserved in the interaction (Curtis and Wilkinson, 1972, Wilson 1977) and the third term is error resulting from the straightahead assumption (Alsmiller et al. 1968, Wilson 1977). The surprising result is that the velocity conserving assumption is inferior to the straightahead approximation for the nearly isotropic space radiation. There are great simplifications using equations (4) and (12) as given below

$$
\boldsymbol{Q} \bullet \boldsymbol{\nabla} \phi_{j}(\boldsymbol{x}, \boldsymbol{O}, E)-A_{j}^{-1} \partial_{E}\left[S_{j}(E) \phi_{j}(\boldsymbol{x}, \boldsymbol{Q}, E)\right]=\sum \sigma_{j k}^{r}(E) \phi_{k}(\boldsymbol{x}, \boldsymbol{O}, E)-\sigma_{j}^{r}(E) \phi_{j}(\boldsymbol{x}, \boldsymbol{Q}, E)
$$

which is strictly applicable to the HZE ions $(Z>2)$. The light ions and neutrons have additional complications arising from the broad energy spectra associated with their production, although the more favorable straightahead approximation is useful as indicated in equation (12) and as found by Alsmiller et al. $(1967,1968)$ in their verification process. The corresponding light ion (and neutron) Boltzmann equation is

$$
\boldsymbol{Q} \bullet \boldsymbol{\nabla} \phi_{j}(\boldsymbol{x}, \boldsymbol{Q}, E)-A_{j}^{-1} \partial_{E}\left[S_{j}(E) \phi_{j}(\boldsymbol{x}, \boldsymbol{Q}, E)\right]=\sum \int \sigma_{j k}^{r}\left(E, E^{\prime}\right) \phi_{k}\left(\boldsymbol{x}, \boldsymbol{Q}, E^{\prime}\right) d E^{\prime}-\sigma_{j}^{r}(E) \phi_{j}(\boldsymbol{x}, \boldsymbol{Q}, E)
$$

where we have made use of the straightahead approximation as given by equation (10). Equations (13) and (14) have sufficient simplicity to allow an approach for both space and laboratory applications. The main force of the laboratory applications allow detailed model testing of the many atomic/molecular and nuclear processes.

\subsection{Marching Procedures}

The first version of the HZETRN code is based on the solution of equations (13) and (14) as guided by the Monte Carlo studies of the straightahead approximation by Alsmiller et al. (1968). We again specialize to solution along a ray $\boldsymbol{Q}$ directed along the x-axis for which equation (14) becomes

$$
\left[\partial_{x}-A_{j}^{-l} \partial_{E} S_{j}(E)+\sigma_{j}^{r}(E)\right] \phi_{j}(x, E)=\sum_{k} \int \sigma_{j k}^{r}\left(E, E^{\prime}\right) \phi_{k}\left(x, E^{\prime}\right) d E^{\prime}
$$


where $\sigma_{j k}^{r}\left(E, E^{\prime}\right)$ are approximated for nucleons by the multiplicities of Ranft (1980), Bertini et al. (1972), and quasi-elastic contribution as described by Wilson et al. (1991). An immediate problem is the near singular nature of the differential operator, and transformation from energy to residual range coordinates as we did in developing the Green's function greatly relieves this problem (Wilson et al. 1991). Unlike the Green's function development, numerical procedures are simplified by introducing only a single residual range coordinate for all ions, and the residual proton range $r$ is used as the common coordinate as

$$
r=\int_{0}^{E} d E^{\prime} / S\left(E^{\prime}\right)
$$

and residual range of other particle types are related as $v_{j} r_{j} \approx r$ with $v_{j}=Z_{j}^{2} / A_{j}$ which fails at low energies corresponding to low residual range due to electron capture into atomic orbitals characteristic to each ion type. The corresponding transport equation is

$$
\left[\partial_{x}-v_{j} \partial_{r}+\sigma_{j}(r)\right] \psi_{j}(x, r)=\Sigma_{k} \int_{r}^{\infty}\left(v_{\mathrm{j}} / v_{\mathrm{k}}\right) s_{j k}\left(r, r^{\prime}\right) \psi_{k}\left(x, r^{\prime}\right) d r^{\prime}
$$

where scaled flux is now ( $v_{\mathrm{j}}$ for neutral particles such as neutrons are taken as unity in scaling relations, Wilson et al. 1991)

$$
\psi_{j}(x, r)=v_{\mathrm{j}} S(E) \phi_{j}(x, E)
$$

and the scaled differential cross sections are

$$
s_{j k}\left(r, r^{\prime}\right)=S(E) \sigma_{j k}^{r}\left(E, E^{\prime}\right)
$$

Errors in scaling of proton stopping and range parameters in arriving at the approximate transport equation (17) are compensated in part by solutions of equation (17) approaching a low energy equilibrium spectrum for ions given by

$$
v_{\mathrm{j}} S(E) \phi_{j}(x, E) \Rightarrow \text { constant }
$$

where the constant is fixed by the higher ion energy. In distinction, the solution to equation (15) for ions has the low energy equilibrium spectrum

$$
A_{j}^{-1} S_{j}(E) \phi_{j}(x, E) \Rightarrow \text { constant }
$$

also fixed by the higher energy flux for which the range scaling relation $v_{j} r_{j} \approx r$ has better validity and the two constants are nearly equal so that equation (21) has improved accuracy over equation (20) at lower energies. This fact will require us to alter the flux unscaling relations as demanded by equation (21) to maintain accuracy at the lower energies. From equations (20) and (21), we can understand the simplicity of numerically solving equation (17) over numerical solution based on equation (15). The solution to equation (17) approaches a constant at small residual ranges allowing large separations in $\mathrm{r}$ grid values with smooth extrapolation to zero range whereas solutions of equation (15) vary as the nearly singular $1 / S_{j}(E)$ for which small $E$ grid spacing is required leading to slow computational procedures. We tested the assumptions in equation (17) and unscaling according to relation (21) later in a separate report (Wilson et al. 2006a).

The confusion caused by different scaling methods and associated coordinates for numerical procedures is justified by the simplification of the numerical representation of fluence of all particle types over a common residual range grid and simplification of the numerical procedures leading to high performance codes. Still a straightforward finite differencing of equation (17) can introduce unstable roots as had plagued the thermal transport problem for many years (Wilson et al. 1991). We will use the unconditionally stable methods of Wilson et al. (1991) arrived at by inverting the differential operator (Wilson et al. 1977, 1989, 1991) of equation (7) as

$$
\psi_{j}(x, r)=\exp \left[-\zeta_{j}(r, x)\right] \psi_{j}\left(0, r+v_{j} x\right)+\Sigma_{k} \int_{0}^{x} \int_{r+v^{j} x^{\prime}} \exp \left[-\zeta_{j}\left(r, x^{\prime}\right)\right]\left(v_{\mathrm{j}} / v_{\mathrm{k}}\right) s_{j k}\left(r+v_{j} x^{\prime}, r^{\prime}\right) \psi_{k}\left(x-x^{\prime}, r^{\prime}\right) d r^{\prime} d x^{\prime}
$$

where the exponential is the integrating factor related to attenuation of the $\mathrm{j}$ type ions with

$$
\zeta_{j}(r, x)=\int_{o}^{x} \sigma_{j}\left(r+v_{j} x^{\prime}\right) d x^{\prime}
$$

and is related to equation (9). Equation (22) is a Volterra equation and can be solved either as a Neumann series (Wilson and Lamkin 1975) or with marching procedures (Wilson et al 1991). Note that the inverse mapping is taken as

$$
\phi_{j}(x, E)=A_{j} \psi_{j}(x, r) / S_{j}(E)
$$

to guarantee the equilibrium solution given as equation (21) at low energies away from the boundaries (note, the proton stopping power is used in case of unscaling the neutron flux). We assume in this treatment that the 
equilibrium constant resulting from equation (22) and given in equation (20) differs little from condition (21) for which the inverse mapping of equation (24) is most accurate. These approximations were tested by Wilson et al. (2006a).

Two tracks are taken in implementing a marching procedure for equation (22) depending on particle type as demanded by the character of the nuclear processes. The problem naturally divides into "light ions" which will refer to all ions with atomic mass of four or less including neutrons and high charge-energy (HZE) ions having atomic mass greater than 4 . The distinction arises from the energy and angle distributions of the double differential cross sections for which the HZE ions leaving a projectile fragmentation event have velocity nearly equal to that of the projectile as approximated by equation (11). Although the light ions are assumed to travel in the same direction as the projectile (see equation 10), they cover a broad energy distribution that cannot be ignored. The marching procedure is obtained by first considering equation (22) evaluated at $x+h$ where $h$ is the step size. Clearly,

$$
\psi_{j}(x+h, r)=\exp \left[-\zeta_{j}(r, h)\right] \psi_{j}\left(x, r+v_{j} h\right)+\Sigma_{k} \int_{0}^{h} \int_{r+v x^{\prime}}{ }^{\circ} \exp \left[-\zeta_{j}\left(r, x^{\prime}\right)\right]\left(v_{\mathrm{j}} / v_{\mathrm{k}}\right) s_{j k}\left(r+v_{j} x^{\prime}, r^{\prime}\right) \psi_{k}\left(x+h-x^{\prime}, r^{\prime}\right) d r^{\prime} d x^{\prime}
$$

which may be used to develop a marching step from $x$ to $x+h$ once a means is found to approximate the field function $\psi_{j}(x, r)$ across the subinterval $\{x, x+h\}$. If $h$ is sufficiently small such that $\sigma_{j}(r) h<<1$ then, following lowest order perturbation theory (Wilson and Lamkin 1975), one has

$$
\psi_{k}\left(x+h-x^{\prime}, r^{\prime}\right)=\exp \left[-\zeta_{k}\left(r^{\prime}, h-x^{\prime}\right)\right] \psi_{k}\left[x, r^{\prime}+v_{k}\left(h-x^{\prime}\right)\right]+O(h)
$$

which may be used to approximate the integral in equation (25) giving results for the fields $O\left(h^{2}\right)$ as required to control the propagated error (Wilson et al. 1991). Substituting equation (26) into (25) and evaluating the attenuation factors at the interval midpoint (mean value theorem) results in

$$
\begin{aligned}
\psi_{j}(x+h, r)= & \left.\exp \left[-\zeta_{j}(r, h)\right] \psi_{j}\left(x, r+v_{j} h\right)+\Sigma_{k} \exp \left[-\zeta_{j}(r, h / 2)-\zeta_{k}\left(r^{\prime}, h / 2\right)\right] \int_{r+v j h / 2}{ }^{\infty}\left(v_{\mathrm{j}} / v_{\mathrm{k}}\right) F_{j k}^{\Delta}\left(h, r, r^{\prime}\right) \psi_{k}\left(x, r^{\prime}+v_{k} h / 2\right)\right] d r^{\prime} \\
& +O\left(h^{2}\right)
\end{aligned}
$$

where the integrand has been simplified using

$$
F_{j k}^{\Delta}\left(h, r, r^{\prime}\right)=\int_{0}^{h} s_{j k}\left(r+v_{j} x^{\prime}, r^{\prime}\right) d x^{\prime}=F_{j k}\left(r+v_{j} h, r^{\prime}\right)-F_{j k}\left(r, r^{\prime}\right)
$$

and

$$
F_{j k}\left(r, r^{\prime}\right)=\int_{0}^{\varepsilon(r)} \sigma_{j k}^{r}\left(E^{\prime \prime}, E^{\prime}\right) d E^{\prime \prime}
$$

with $\varepsilon(r)$ the energy associated with proton residual range $r$, and $E^{\prime}=\varepsilon\left(r^{\prime}\right)$. Note that if $j$ corresponds to a neutral particle such as the neutron $(j=n)$ then the above expressions are evaluated in the limit as $v_{j}$ approaches zero in the range scaling relations resulting in the following (whereas the flux scaling factor for neutrons assumes $v_{n}=1$ )

$$
\begin{gathered}
\psi_{n}(x+h, r)=\exp \left[-\sigma_{n}(r) h\right] \psi_{n}(x, r)+\sum_{k \neq n} \exp \left[-\sigma_{n}(r) h / 2-\zeta_{k}\left(r^{\prime}, h / 2\right)\right] h \int_{r}^{\infty}\left(1 / v_{k}\right) s_{n k}\left(r, r^{\prime}\right) \psi_{k}\left(x, r^{\prime}+v_{k} h / 2\right) d r^{\prime} \\
+\exp \left[-\sigma_{n}(r) h / 2-\sigma_{n}\left(r^{\prime}\right) h / 2\right] h \int_{r}^{\infty} s_{n n}\left(r, r^{\prime}\right) \psi_{n}\left(x, r^{\prime}\right) d r^{\prime}
\end{gathered}
$$

and similarly for the neutral $k$ term $(k=n)$ when the $j$ particle is charged

$$
\begin{aligned}
\psi_{j}(x+h, r)= & \exp \left[-\zeta_{j}(r, h)\right] \psi_{j}\left(x, r+v_{j} h\right)+\Sigma_{k \neq n} \exp \left[-\zeta_{j}(r, h / 2)-\zeta_{k}\left(r^{\prime}, h / 2\right)\right] \int_{r}^{\infty}\left(v_{\mathrm{j}} / v_{\mathrm{k}}\right) F_{j k}^{\Delta}\left(h, r, r^{\prime}+v_{j} h / 2\right) \\
& \left.\times \psi_{k}\left[x, r^{\prime}+\left(v_{j}+v_{k}\right) h / 2\right)\right] d r^{\prime}+\exp \left[-\zeta_{j}(r, h / 2)-\sigma_{n}\left(r^{\prime}\right) h / 2\right] \int_{r}^{\infty} v_{\mathrm{j}} F_{j n}^{\Delta}\left(h, r, r^{\prime}+v_{j} h / 2\right) \psi_{n}\left(x, r^{\prime}+v_{j} h / 2\right) d r
\end{aligned}
$$

where $v_{n}$ in the flux scaling relation (24) is taken as unity. Equations (30) and (31) are solved on an equal-spaced $x$ grid $\Delta x=h$ apart and a logarithmic spaced $r$-grid on two subintervals. The remaining integrals in these equations are approximated by (Wilson et al 1991)

$$
\int_{r k}^{\infty} K\left(r_{n}, r^{\prime}\right) \psi_{j}\left(x, r^{\prime}\right) d r^{\prime} \approx \sum_{l=k}^{\infty} K\left[r_{n},\left(r_{l}+r_{l+l}\right) / 2\right] \int_{r l}^{r l+1} \psi_{j}\left(x, r^{\prime}\right) d r^{\prime}
$$

where $\infty$ denotes a chosen upper limit tailored to the specific boundary condition. Note that the matrix of $K$-values can be evaluated once on the $r$-grid and stored for subsequent steps providing high computational efficiency. Equations (30) and (31) provide the basis for the light ion transport of the HZETRN code. The HZE ion projectile $\left(A_{j}>4\right)$ coupling to the light fragments is contained in equations (27) to (31).

The HZE fragments are produced with nearly the same velocity as the projectile ion as expressed in equation (13) and results in the simplified Boltzmann equation as

$$
\left[\partial_{x}-A_{j}^{-1} \partial_{E} S_{j}(E)+\sigma_{j}^{r}(E)\right] \phi_{j}(x, E)=\sum_{k} \sigma_{j k}^{r}(E) \phi_{k}(x, E)
$$

for which the scaled equations result in contributions from all HZE ions (with $A_{k}>4$ ) as 


$$
\psi_{j}(x, r)=\exp \left[-\zeta_{j}(r, x)\right] \psi_{j}\left(0, r+v_{j} x\right)+\Sigma_{k} \int_{0}^{x} \exp \left[-\zeta_{j}\left(r, x^{\prime}\right)\right]\left(v_{\mathrm{j}} / v_{\mathrm{k}}\right) \sigma_{j k}^{r}\left(r^{+}+v_{\mathrm{j}} x^{\prime}\right) \psi_{k}\left(x-x^{\prime}, r+v_{\mathrm{j}} x^{\prime}\right) d x^{\prime}
$$

The corresponding marching equation (Wilson et al. 1991, Shinn et al. 1992) is given as

$$
\psi_{j}(x+h, r)=\exp \left[-\zeta_{j}(r, h)\right] \psi_{j}\left(x, r+v_{j} h\right)+\Sigma_{k} \int_{0}^{h} \exp \left[-\zeta_{j}\left(r, x^{\prime}\right)\right]\left(v_{\mathrm{j}} / v_{\mathrm{k}}\right) \sigma_{j k}\left(r+v_{j} x^{\prime}\right) \psi_{k}\left(x+h-x^{\prime}, r+v_{j} x^{\prime}\right) d x^{\prime}
$$

for which the integrand can be approximated for sufficiently small $h$ using

$$
\psi_{k}\left(x+h-x^{\prime}, r_{+} v_{j} x^{\prime}\right)=\exp \left[-\xi_{k}\left(r_{+} v_{j} x^{\prime}, h-x^{\prime}\right)\right] \psi_{k}\left[x, r_{+} v_{j} x^{\prime}+v_{k}\left(h-x^{\prime}\right)\right]+O(h)
$$

allowing the following simplification

$$
\begin{aligned}
\psi_{j}(x+h, r)= & \exp \left[-\zeta_{j}(r, h)\right] \psi_{j}\left(x, r+v_{j} h\right)+\Sigma_{k} \int_{0}^{h} \exp \left[-\zeta_{j}\left(r, x^{\prime}\right)-\zeta_{k}\left(r_{+} v_{j} x^{\prime}, h-x^{\prime}\right)\right]\left(v_{\mathrm{j}} / v_{\mathrm{k}}\right) \\
& \times \sigma_{j k}\left(r+v_{j} x^{\prime}\right) \psi_{k}\left[x, r_{+} v_{j} x^{\prime}+v_{k}\left(h-x^{\prime}\right)\right] d x^{\prime}
\end{aligned}
$$

To evaluate equation (37), we use the mean value theorem that guarantees linear terms of the final integral to be zero. First, we expand the attenuation factor as

and similarly for

$$
\zeta_{j}\left(r, x^{\prime}\right)=\int_{o}^{x^{\prime}} \sigma_{j}\left(r_{+} v_{j} x^{\prime \prime}\right) d x^{\prime \prime} \approx \int_{o}^{x^{\prime}}\left[\sigma_{j}\left(r_{+} v_{j} h / 2\right)+\partial_{r} \sigma_{j}\left(r_{+} v_{j} h / 2\right) v_{j}\left(x^{\prime \prime}-h / 2\right)\right] d x^{\prime \prime}
$$

$$
\begin{aligned}
\zeta_{k}\left(r+v_{j} x^{\prime}, h-x^{\prime}\right) & =\int_{o}^{h-x^{\prime}} \sigma_{k}\left[r_{+} v_{j} x^{\prime}+v_{k}\left(h-x^{\prime \prime}\right)\right] d x^{\prime \prime} \approx \int_{o}^{h-x^{\prime}}\left[\sigma_{j}\left(r_{+} v_{j} x^{\prime}+v_{k} h / 2\right)\right. \\
& \left.+\partial_{r} \sigma_{j}\left(r_{+} v_{j} x^{\prime}+v_{k} h / 2\right) v_{k}\left(x^{\prime \prime}-h / 2\right)\right] d x^{\prime \prime}
\end{aligned}
$$

while applying the mean value theorem to the remaining factors of equation (37) and neglecting all but linear expansion terms in the integrand yields

$$
\begin{aligned}
& \psi_{j}(x+h, r)=\exp \left[-\zeta_{j}(r, h)\right] \psi_{j}\left(x, r+v_{j} h\right)+\Sigma_{k}\left(v_{\mathrm{j}} / v_{\mathrm{k}}\right) \sigma_{j k}\left(r+v_{j} h / 2\right) \psi_{k}\left[x, r_{+}\left(v_{j}+v_{k}\right) h / 2\right] \\
&\left.\times \int_{0}^{h} \exp \left\{-\sigma_{j}\left(r+v_{j} h / 2\right) x^{\prime}-\sigma_{k}\left[r_{+}\left(v_{j}+v_{k}\right) h / 2\right)\left(h-x^{\prime}\right)\right]\right\} d x^{\prime} \\
&=\exp \left[-\zeta_{j}(r, h)\right] \psi_{j}\left(x, r+v_{j} h\right)+\Sigma_{k}\left(v_{\mathrm{j}} / v_{\mathrm{k}}\right) \sigma_{j k}\left(r+v_{j} h / 2\right) \psi_{k}\left[x, r_{+}\left(v_{j}+v_{k}\right) h / 2\right] \\
&\left.\left.\times\left[\exp \left\{-\sigma_{j}\left(r_{+} v_{j} h / 2\right) h\right\}-\exp \left\{-\sigma_{k}\left[r_{+}\left(v_{j}+v_{k}\right) h / 2\right)\right] h\right\}\right] /\left\{\sigma_{k}\left[r_{+}\left(v_{j}+v_{k}\right) h / 2\right)\right]-\sigma_{j}\left(r_{+} v_{j} h / 2\right)\right\}+O\left(h^{2}\right)
\end{aligned}
$$

to be compared with the original HZETRN algorithm to $O\left[\left(v_{j}-v_{k}\right) h\right]$ derived by Shinn et al. (1992) given as

$$
\psi_{j}(x+h, r) \approx \exp \left[-\zeta_{j}(r, h)\right] \psi_{j}\left(x, r+v_{j} h\right)+\Sigma_{k}\left(v_{\mathrm{j}} / v_{\mathrm{k}}\right) \sigma_{j k}(r) \psi_{k}\left(x, r+v_{j} h\right)\left\{\exp \left[-\sigma_{j}(r) h\right]-\exp \left[-\sigma_{k}(r) h\right]\right\} /\left[\sigma_{k}(r)-\sigma_{j}(r)\right]
$$

In earlier versions of BRYNTRN for proton/neutron transport, the flux scaling relation was taken correctly as

$$
\psi_{j}(x, r)=S(E) \phi_{j}(x, E)
$$

but carried over to the final BRYNTRN for light-ions/neutron transport in the most recent version of BRYNTRN (Cucinotta 1993). In coupling to HZETRN with scaling given by

$$
\psi_{j}(x, r)=v_{\mathrm{j}} S(E) \phi_{j}(x, E)
$$

there is an inconsistency in flux scaling which must be accounted for. The appropriate coupling is given in equations (37) through (41) with the added factor of $v_{\mathrm{j}} / v_{\mathrm{k}}$ in the field coupling terms. The main effects on solution of the Boltzmann equation are expected for the light ions of $\mathrm{H}^{2}, \mathrm{H}^{3}$, and $\mathrm{He}^{3}$ with only minor effects on the major light-ion/neutron components $\left(\mathrm{n}, \mathrm{H}^{1}, \mathrm{He}^{4}\right)$. Note the main difference in the original and corrected code is determined by the ratios of $v_{\mathrm{j}} / v_{\mathrm{k}}$ of the field coupling terms. Current code verification processes are discussed in Wilson et al. (2006a).

\section{LEO Environmental Model}

The LEO environment consists of three main sources. Galactic cosmic rays (GCR) that penetrate the geomagnetic field, albedo neutrons from GCR interaction with the Earth's atmosphere, and particles trapped in the geomagnetic field. Three primary limitations in the environmental models are that the AP8 MIN\&MAX are time/direction independent and that the vertical geomagnetic cutoff is used to describe the transmitted galactic cosmic rays. These models have been relatively successful in describing the radiation environment aboard the highly maneuverable Shuttle wherein anisotropies tend to be averaged. Such models will not be adequate for the ISS mainly oriented in the local horizontal plane along the velocity vector (minimum drag) except during battery charging. In a previous paper, we developed a dynamic/anisotropic trapped proton environment and general 
geomagnetic cutoff model (Wilson et al. 2006b). These models are placed in a suitable form for evaluation of the incident radiation on the bounding surface of the six-degree of freedom motion of an orbiting spacecraft for shield evaluation.

\subsection{Albedo Neutrons}

Albedo neutrons result from the interaction of cosmic rays with the Earth's atmosphere. As the cosmic ray intensities are modulated by solar activity so are the atmospheric neutrons modulated with time. The atmospheric neutron model is a parametric fit to data gathered by Langley Research Center studies of the radiations at SST altitudes in the years 1965 to 1971 covering the rise and decline of solar cycle 20. Scaling of the data with respect to geomagnetic cutoff, altitude, and modulation of the Deep River Neutron Monitor was found to allow mapping of the environment to all locations at all times resulting in an empirically based model for atmospheric neutrons (Wilson et al. 1991). The leakage flux $F_{L}(E)$ is closely related to the differential flux $\phi(E, \Omega)$ at the top of the atmosphere as follows

$$
F_{L}(E)=\int \phi(E, \Omega) \cos \theta d \Omega
$$

where $\cos \theta$ is the direction cosine of the velocity vector with the zenith (note, $\phi(E, \Omega)=0$ for $\cos \theta<0$ ). There are unresolved differences among various measurements of the leakage flux that is in part the assumed angular dependence of the differential flux.

Table 1. Ratio of leakage flux to integrated flux as a function of energy and geomagnetic cutoff.

\begin{tabular}{|c|c|c|c|c|c|c|}
\hline $\mathrm{P}_{\mathrm{c}}, \mathrm{GV}$ & \multicolumn{5}{|c|}{ Leakage to integrated flux ratio for energy $(\mathrm{MeV})$ of- } \\
\hline & $<.001$ & $.001-1$ & $1-10$ & $10-19$ & $19-100$ & $100-2000$ \\
\hline 0 & 0.07 & 0.63 & 0.55 & 0.48 & 0.45 & $0.38-0.23$ \\
\hline 17 & 0.07 & 0.64 & 0.56 & 0.49 & 0.53 & $0.46-0.39$ \\
\hline
\end{tabular}

The ratio of leakage flux to integrated flux is given by the New York University (NYU) group (Korff et al. 1979) in Table 1 that we use in the present model. The leakage flux was measured on the OGO-6 satellite by the University of New Hampshire (Lockwood 1972) during June 1969. The leakage flux spectrum was measured (Jenkins et al. 1971, Preszler et al. 1972) and calculated (Korff et al. 1979) by various groups over Palestine, Texas during September 1971 and approximated by Wilson et al. (1999) as

$$
F_{L}(E)=\left\{\begin{array}{l}
0.065 / E \text { for } E \leq 10 \mathrm{MeV} \\
0.0026 \exp (-0.011 E) \text { for } E>10 \mathrm{MeV}
\end{array}\right.
$$

The total leakage flux is shown in Fig. 3 at various solar maxima and minima and in particular with the measurements on the OGO-6 satellite during June 1969 which was a local maximum of cycle 20 (i.e., local minimum of DRNM). The leakage flux at the top of the atmosphere is extrapolated to low Earth orbit altitude according to Gauss' law (varies as $\mathrm{r}^{-2}$ ). There are some inconsistencies among various groups on specific neutron field related quantities. The present model is biased to the Palestine spectrum of September 1971, the OGO-6 latitude dependence during the local DRNM minimum of June 1969, and solar cycle dependence of the high altitude atmospheric neutron measurements as is clear from the above discussion (Wilson et al. 2002b).

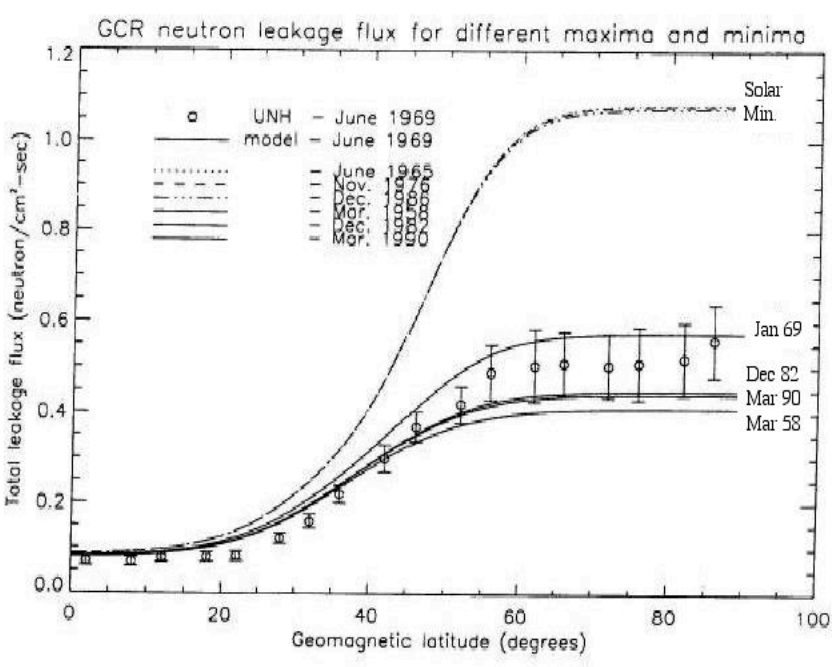

Fig. 3 GCR induced neutron leakage flux for different solar cycle maxima and minima compared to measurements by Univ. New Hampshire (Lockwood et al. 1972)

\subsection{Trapped Proton Environment}

The trapped proton population is modeled as AP8 for 1965 solar minimum and 1970 solar maximum (Vette 1991). These inner zone particles are injected through the decay of albedo neutrons as they leak from the Earth's atmosphere into the magnetic bottle defined by the Störmer forbidden zones (Wilson 2000). The inner zone 
particles are lost from the trapping region by interaction with the tenuous atmosphere and generally have long trapping lifetimes above the fringes of the atmosphere. The inner zone consists of both proton and electron decay products. The average kinetic energy of the inner zone electrons is a few hundred keV. The electrons are easily removed from the spacecraft interior by the slightest amount of shielding and are mainly of concern to an astronaut in a spacesuit and materials external to the spacecraft. Within any pressure vessel such as has the Shuttle or ISS, the electrons are easily shielded by the micrometeoroid/debris bumper and pressure vessel wall. Of the trapped particles, only the protons with energies near or above one hundred $\mathrm{MeV}$ are of concern to the interior environment of the Shuttle or ISS.

The particles trapped in the geomagnetic field were modeled from data obtained during two epochs of solar cycle 20 (solar minimum of 1965 and "solar maximum" of 1970) and are used with the geomagnetic fields on which the B/L maps were prepared (McCormack 1988). The 1965 analysis using the magnetic field model of Jensen and Cain (1962) resulted in particle population maps AP8 MIN (Sawyer and Vette 1976). The 1970 analysis using the magnetic field model GSFC 12/66 (Cain et al. 1967) extrapolated to 1970 resulted in the particle population maps of AP8 MAX (Sawyer and Vette 1976). These models are considered the best available global representations of the trapped proton environment. This includes the known uncertainties in the AP8 models of a factor of 2 in LEO applications.

It was believed at one time that better estimates of particle environments could be gained by evaluating the population maps defined on invariant McIlwain coordinates over current magnetic field conditions. This interpolation would, for example, contain the westward drift of the South Atlantic Anomaly (SAA) observed in recent years by Badhwar et al. (1996). However, it was recognized by the Shuttle dosimetry group (Atwell et al. 1989) that large errors resulted from such a procedure that does not account accurately the altitude shifts with changing magnetic fields. It was concluded that the use of the particle population maps interpolated over the magnetic field model for which the population map was derived would provide the best estimates of the long-term orbital averaged particle environments even though the westward drift is not represented. The westward drift is often introduced as a rotation of geographic coordinates $(0.3$ degree/yr) without regard to modifying the magnetic field over which the AP8 models were derived (Heynderickx 1996).

In a prior report (Wilson et al. 2006b), we used the conventional westward drift of 0.3 degrees with an initial realignment of location such that AP8 MIN and MAX would be centered in 1965 and drift together 0.3 degrees per year into the future with an improved modulation function. A renewed analysis of drifting of fields will be addressed in a companion paper wherein the spatial distribution of the radiation is addressed. The present paper will address long-term averages in which field drift is unimportant (Atwell et al. 1989) and focus on re-evaluation of the modulation factor and angular dependence.

The proton omni-directional flux spectrum at any location and time $f_{p}(r, \theta, \phi, E, t)$ is then extrapolated using the following functional form of Wilson et al. (1999, 2006b, see also Blanchard and Hess 1965)

$$
f_{p}(r, \theta, \phi, E, t)=f_{p, \text { min }}(r, \theta, \phi, E) \exp \left\{-\alpha_{p}\left[D R N M \times F_{10.7}-D R N M \times F_{10.7} I_{M I N}\right]\right\}
$$

where $f_{p, \min }(r, \theta, \phi, E)$ is the proton flux at solar minimum shifted to time $t$ and $\alpha_{p}$ is evaluated using the solar maximum $f_{p, \max }(r, \theta, \phi, E)$ related to AP8 MIN\&MAX models but with the latitude and longitude tentatively shifted after centering in 1965 and 1970 as discussed by Wilson et al. (2007). In equation (46), the quantity $\left(D R N M \times F_{10.7}\right)$ is averaged over the prior 14 months at solar minimum and 2 months at solar maximum with linear interpolation as determined by a best fit (Fig. 4) to the limited NOAAPRO model (Huston and Pfitzer 1998). Following the analysis of Wilson et al. (2007), we use the proton flux at solar minimum with

$f_{p, \min }(r, \theta, \phi, E)=0.5 f_{\text {APSMIN }}(r, \theta-2.4-0.07 \Delta t, \phi+4.1+0.19 \Delta t, E)$

and solar maximum with

$f_{p, \max }(r, \theta, \phi, E)=0.6 f_{A P S M A X}(r, \theta-4.8-0.07 \Delta t, \phi+8+0.19 \Delta t, E)$

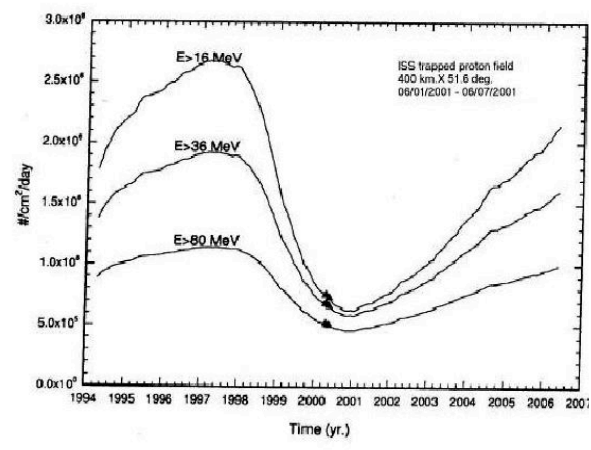

Fig. 4 Trapped proton environment in ISS orbit during June 2001.

where $\Delta t$ is time after the map epoch. The sunspot numbers and Deep River neutron monitor variation over solar cycle 23 (observed and projected) are used (Wilson et al. 2002b, Kim et al. 2006).

Heckman and Nakano (1963) had studied the angular distribution of trapped protons with nuclear emulsion on rockets many years ago and presented a simple model of the pitch angle distributions about the geomagnetic field lines as related to the lifetimes of particles with guiding centers on different field lines. The protons' velocity 
vectors lie within 15 degrees of a plane perpendicular to the geomagnetic field line as seen in Fig. 5 . Thus, those protons arriving from the east or the west differ in intensity according to the atmosphere scale height as related to the difference in population lifetime. The directional intensity is then defined for direction of arrival given by the local pitch angle $\theta$ and azimuth $\lambda$ distribution as follows

$$
\frac{j(\theta, \lambda)}{J_{4 \pi}}=C \exp \left[\frac{-(\pi / 2-\theta)^{2}}{2 \sigma_{\theta}{ }^{2}}\right] \exp \left[\frac{r_{g} \cos I \cos \lambda}{h_{s}}\right]
$$

where $r_{g}$ is the particle gyro-radius about the field line, I is the dip angle, $h_{s}$ is the atmospheric scale height, $\sigma_{\theta}$ is the width of the pitch angle distribution, and $\mathrm{C}$ is a normalization constant as discussed by Kern (1994). It was shown by Heckman and Nakano (1963) that $\sigma_{\theta}$ depends on atmospheric scale height, altitude, and dip angle so that pitch angle distributions are nearly independent of particle energy. In distinction, the east-west asymmetry depends on the particle gyro-radius displaying marked energy dependence. The International Geophysical Reference Field (IGRF) is implemented at the current time and the scale height is found from the solar modulated fit of Pfitzer (1990) used by Badhwar (1999) to organize the Shuttle dosimetry data and given as

$$
\rho(\mathrm{r})=\rho_{0} \exp \left\{-(\mathrm{h}-120) /\left[\mathrm{A}(\mathrm{h}-103)^{1 / 2}\right]\right\}
$$

where $\rho_{0}=2.7 \times 10^{-11} \mathrm{~g} / \mathrm{cm}^{3}, \mathrm{~h}$ is altitude in $\mathrm{km}$, and fitting parameter $\mathrm{A}=0.99+0.518[(\mathrm{~F}+\mathrm{Fbar}) / 110]^{1 / 2}$ with Fbar the average of $\mathrm{F}$ given as the F10.7 solar radio output parameter over three prior solar rotations ( 81 days). The trapped protons are encountered by LEO spacecraft in the SAA, and ISS encounters this region from two directions (first with ascending node and second with descending node) as occurs during orbit precession. In addition, the radiation incident on the outer surface of the spacecraft is required for shield evaluation, and the attitude of the spacecraft is never fixed but limit cycles if not under reorientation from required maneuvers. The angular distribution averaged over spacecraft attitude in the region of radiation encounter is then to be evaluated. This is accomplished by relating the orientations in the spacecraft frame through yaw plus heading, pitch, and roll to the local vertical reference frame where the radiation environment is evaluated. We normally use 970 ray directions to evaluate the boundary conditions for shield evaluation and will use these same directions for evaluation of the directional environment.

\subsection{Geomagnetic Transmission Factor}

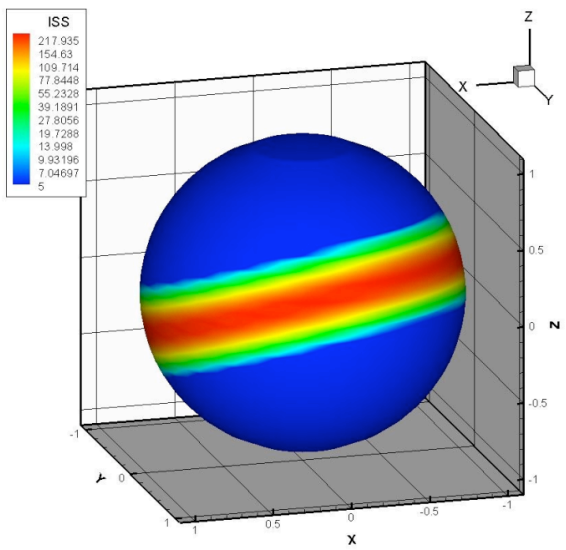

Fig. 5 Angular distribution of $82 \mathrm{MeV}$ protons near center of South Atlantic Anomaly.

In the past, the geomagnetic transmission factor used was based on extrapolation of a world map of vertical cutoff rigidities of Smart and Shea (1983). In this model, it was assumed that there is no transmission below the vertical cutoff and 100 percent transmission (excepting the Earth's shadow) above the vertical cutoff. There is, in fact, variable transmission dependent on angle of incidence relative to the east direction. The directional dependent cutoff for a centered dipole field is given by Störmer theory in terms of the cutoff rigidity as

$$
\mathrm{P}_{\mathrm{c}}(\mathbf{Q})=\mathrm{M} \cos ^{4}(\lambda) /\left\{\mathrm{r}^{2}\left[1+\left(1-\cos ^{3}(\lambda) \cos \left(\omega_{\mathrm{E}}\right)\right]^{2}\right\}\right.
$$

where $\mathbf{Q}$ is the local direction of incidence, $\mathbf{M}$ is the dipole moment, $\lambda$ the magnetic latitude, $\cos \left(\omega_{\mathrm{E}}\right)$ is the projection of $\mathbf{Q}$ on the eastward direction. In application to the geomagnetic field, one must first transform the geocentric location into eccentric dipole coordinates. It was shown by Quenby and Webber (1959) that higher multipole contributions can be approximated by replacing $\lambda$ of equation (51) with $\lambda$ ' given by

$$
\lambda^{\prime}=\tan ^{-1}\left\{\left[\mathrm{~V}_{\mathrm{c}}+0.52 \delta_{\mathrm{v}}\right] /\left[2\left(\mathrm{H}_{\mathrm{c}}+0.52 \delta_{\mathrm{H}}\right)\right]\right\}
$$

where $\mathrm{V}_{\mathrm{c}}$ and $\mathrm{H}_{\mathrm{c}}$ are the vertical and horizontal field components at the location and $\delta_{\mathrm{V}}$ and $\delta_{\mathrm{H}}$ field deviations from dipole values.

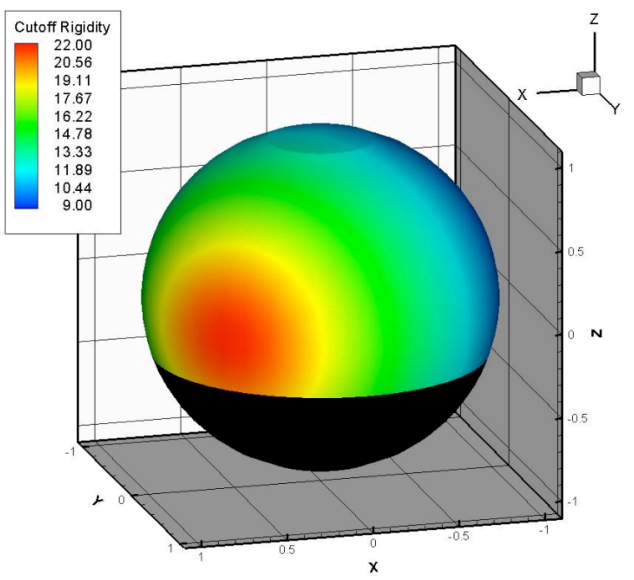

Fig. 6 Angular distribution of geomagnetic cutoffs at the equator near SAA. 
The Quenby/Webber model is of itself rather inaccurate (up to 25 percent) but a renormalization of the transmission factor (along the vertical $\cos \left(\omega_{\mathrm{E}}\right)=0$ ) using the more accurate vertical cutoffs as evaluated by Smart and Shea allows reasonably accurate anisotropic cutoff values (De Angelis et al. 2003). One result of the new transmission factors will be the admission of lower energy cosmic rays than in the currently used vertical cutoff model. In the present model, we use the IGRF field model evaluated for arbitrary dates from 1945 to 2020 . A typical transmission factor of the model is shown in Fig. 6 at a location on the equator over the Earth near the SAA at 400 $\mathrm{km}$ in March 1995. The western approaches are seen as the high cutoff regions on the sphere. The cosmic ray model is that of Badhwar-O'Neill (1995) extrapolated according to neutron monitor count rates (Wilson 2006b).

\section{Model Validation}

Model validation has generally used Shuttle measurements with TLD100 to examine the long-term modulation of the trapped environment with more detailed studies with ISS in which the angular variations have some importance (Hugger et al. 2003, Wilson et al. 2006b). The present step of model validation will focus on the ISS DOSMAP Experiment (E094, PI: Dr. Günther Reitz, DLR) consisting of passive thermo-luminescent detectors (TLD), passive nuclear track detector packages (NTDPs), active dosimeter telescopes (DOSTELs), and mobile dosimeter units (MDUs, identified as Liulin-E094, PI: Dr. T. Dachev 2006). The Liulin energy loss spectra have been correlated to the NTDP derived liner enetgy transfer (LET) spectra by dividing the MDU energy loss spectra by the Liulin detector thickness of $0.306 \mathrm{~mm} \mathrm{Si}$ and conversion to equivalent water. Unlike the TLD and NTDP data (Benton 2004), the advantage of the active detectors is their temporal variations used to separate

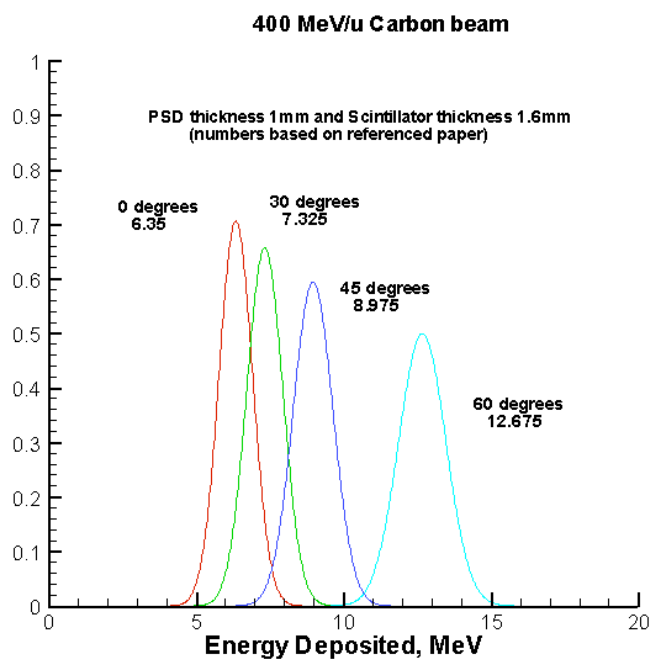

Fig. 7 Liulin detector response model for incident $400 \mathrm{~A} \mathrm{MeV}$ carbon beam.

SAA and GCR contributions. The Liulin energy loss spectral response functions can be accurately modeled as a function of incident angle, particle type, mean energy loss, and energy loss fluctuations due to straggling (Wilson et al. 2002). The response for $\mathrm{C}-12$ ions at $400 \mathrm{~A} \mathrm{MeV}$ is given in Fig. 7 and compares favorably with the measurements using the HIMAC in Chiba (Uchihori et al. 2002).

The data were recorded aboard ISS 6A during the late June - early July time frame of 2001 for several locations in the US Lab Module and Node 1 at 30second time intervals. The positional trajectory data were supplied along with the time sequential measurements. Table 2 denotes pertinent information for the data sets analyzed in the present work.

Table 2. Descriptive parameters for LIULIN data sets.

\begin{tabular}{|c|c|c|c|c|}
\multicolumn{1}{|c}{ Designation } & $\begin{array}{c}\text { Dates of } \\
\text { Measurement }\end{array}$ & $\begin{array}{c}\text { Measurement } \\
\text { Time Span, hr }\end{array}$ & \multicolumn{2}{c|}{$\begin{array}{c}\text { MDU } \\
\text { Number of } \\
\text { Data Records }\end{array}$} \\
\hline SET-1 & July 6-9, 2001 & 82.13 & 2 & 9856 \\
\hline SET-2 & July 6-13, 2001 & 138.60 & 1 & 16633 \\
\hline SET-3 & July 6-13, 2001 & 138.55 & 3 & 16625 \\
\hline SET-4 & June 26-28, 2001 & 57.46 & 1 & 6893 \\
\hline
\end{tabular}

The recently-developed NASA-Langley space radiation environment code (GEORAD), described elsewhere (Wilson et al. 2006b, 2007), has been utilized to simulate the environments for the orbiting ISS for the
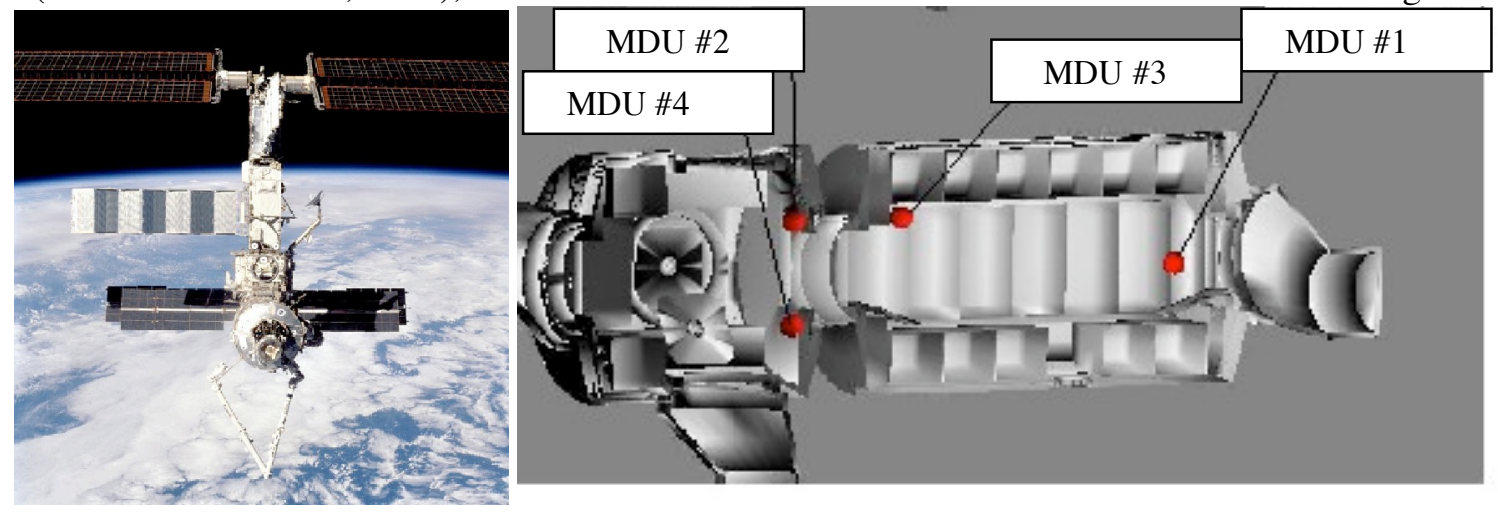

Fig. 8. ISS photo in 6A configuration and CAD-modeled Lab Module with detector locations. 
time periods and the orbital tracks associated with each data set. During the measurement period, ISS was in its designated 6A development stage shown in Fig. 8 with the CAD model simulation.

The CAD model utilizes the I-DEAS ${ }^{\circledR}$ commercial software package and consists of 512 individual components (including detector parts) specified by their spatial location and effective density (mass/volume). The established CAD model then enables the construction of a directional thickness distribution about each target point. Finally, with a given environment (trapped protons and GCR) and an associated shield distribution, the NASALangley transport code 2005 HZETRN is utilized to predict particle flux and dosimetric quantities at each target point indicated in the figure using the E094 site designations.

\subsection{Mission Segment Data}

Sample records of relevant data (formatted as ASCII values) are given in Table 3 as part of the information relating to sequential time points along the orbital track. Note that the exposures have been expressed in terms of a dose rate $(\mu \mathrm{Gy} / \mathrm{hr})$. The general nature of the high quality and consistency of the data is shown in Fig. 9 where the first two days of data set 2 are shown as measured dose rate as a function of time (corresponding to MDU-1 located at the TLD103 site in Fig. 8).

The repetitive low-level ripple is indicative of the GCR exposures, while the sharp "spikes" represent passage through the SAA. In order to extract the SAA trapped proton dose from the underlying GCR, an

Table 3. Sample Data Records from LIULIN Data Set

\begin{tabular}{|c|c|c|c|c|c|}
\hline $\begin{array}{l}\text { Elapsed Time } \\
(\mathrm{hr})\end{array}$ & $\begin{array}{c}\text { Dose Rate } \\
\mu \mathrm{Gy} / \mathrm{hr}\end{array}$ & $\begin{array}{l}\text { Latitude } \\
(\operatorname{deg} N)\end{array}$ & $\begin{array}{l}\text { Longitude } \\
\text { (deg E) }\end{array}$ & $\begin{array}{c}\text { Altitude } \\
(\mathrm{km})\end{array}$ & $\begin{array}{c}\text { Heading } \\
\text { (deg) }\end{array}$ \\
\hline$*$ & $*$ & $*$ & $*$ & $*$ & $*$ \\
\hline * & * & $*$ & $*$ & * & $*$ \\
\hline 19.0500 & 3.41 & -43.65 & 294.01 & 390.36 & 65.14 \\
\hline 19.0583 & 2.92 & -42.62 & 296.15 & 390.21 & 63.49 \\
\hline 19.0667 & 3.77 & -41.55 & 298.22 & 390.05 & 61.66 \\
\hline 19.0750 & 3.99 & -40.43 & 300.21 & 389.88 & 59.93 \\
\hline 19.0833 & 7.92 & -39.28 & 302.14 & 389.71 & 58.24 \\
\hline 19.0917 & 16.62 & -38.09 & 303.99 & 389.52 & 56.56 \\
\hline 19.1000 & 26.74 & -36.87 & 305.79 & 389.34 & 55.02 \\
\hline 19.1083 & 45.20 & -35.62 & 307.52 & 389.15 & 53.35 \\
\hline$*$ & $*$ & $*$ & $*$ & $*$ & $*$ \\
\hline$*$ & $*$ & $*$ & $*$ & $*$ & $*$ \\
\hline
\end{tabular}
analysis script was written that effectively filters the SAA "spikes" and defines the time interval for SAA passage. A sinusoidal function has been used to fit the smoothed low-level GCR for the time period and subtracted from the measured total to obtain the SAA exposure for a single pass. An illustration of this procedure is shown in Fig. 10, where the dose rate data corresponding to an SAA pass (first "spike" of Fig. 9) is plotted over the appropriate time interval. The fit for underlying GCR is also shown.

MDU-1 Data (6-13 Jun 01)

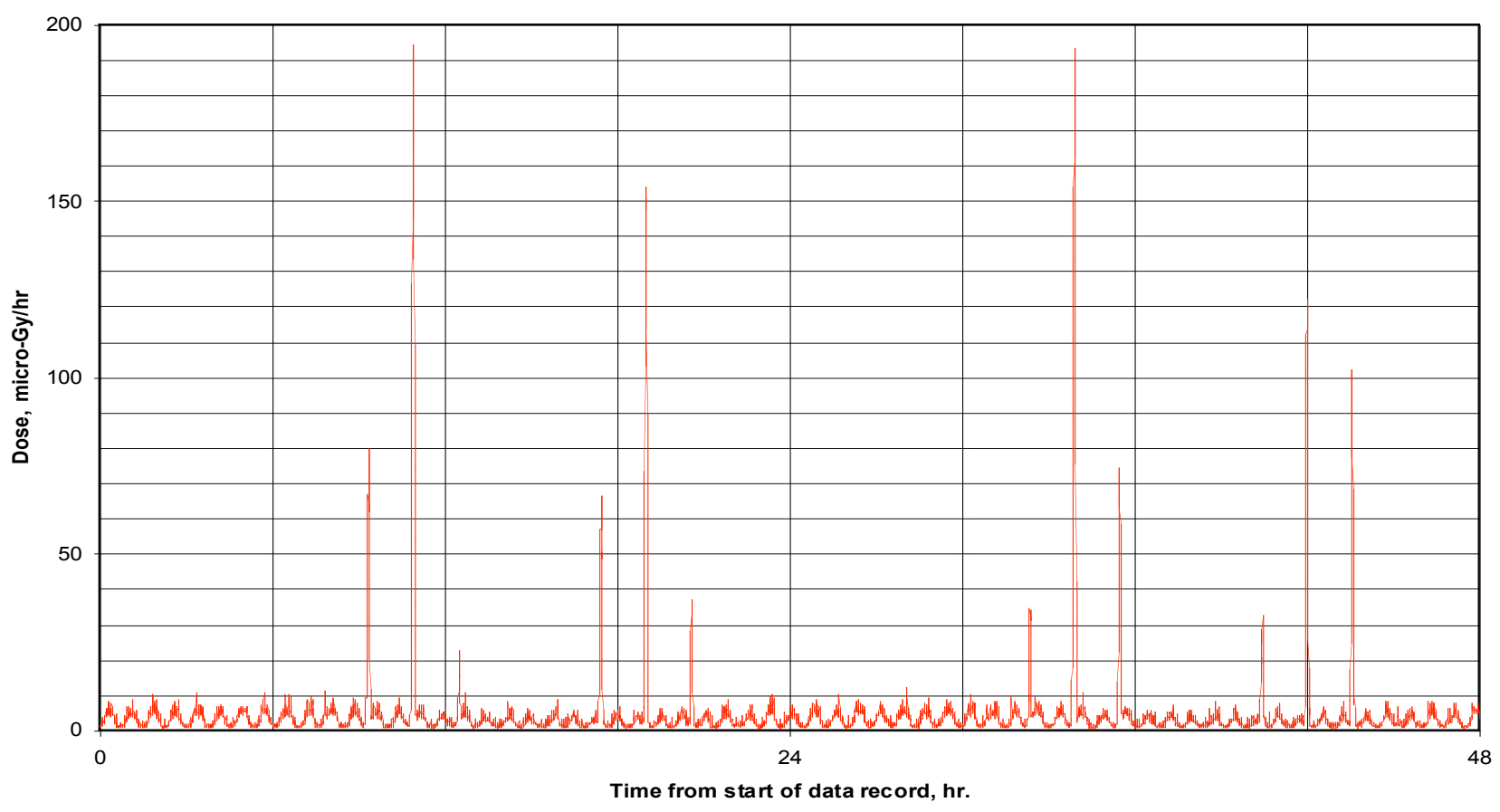

Fig. 9. Sample data from LIULIN detector. 


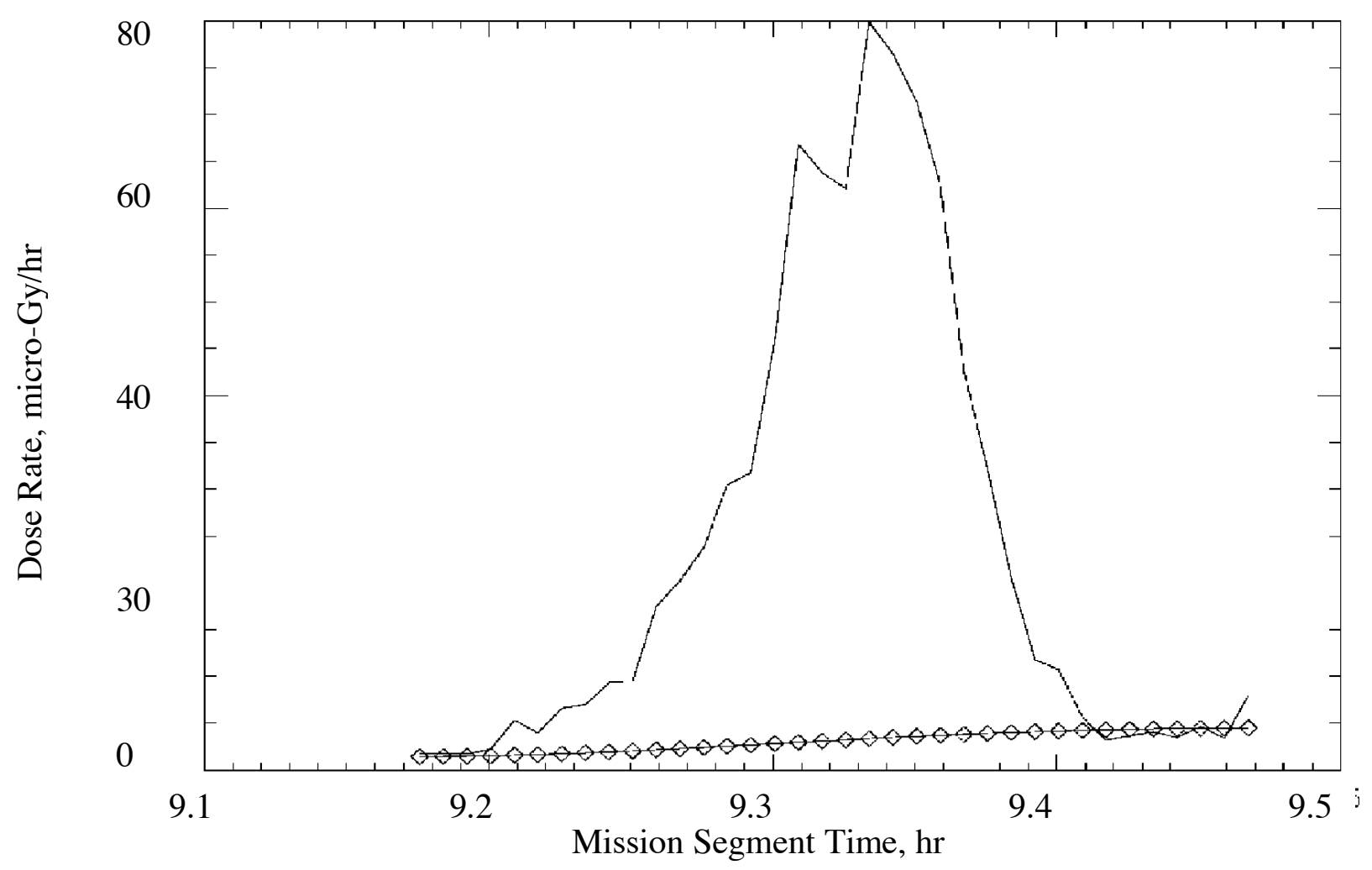

Fig. 10. Sample dose rate data for single pass through SAA with associated underlying GCR.

The total mission segment dose is obtained by direct integration of the dose rate data with respect to time. The corresponding integrations of the individual SAA passes after GCR subtraction render the mission segment exposure for trapped protons. Finally, subtraction of the trapped contribution from the total segment dose results in the GCR dose. A summary of the measured data thus analyzed is given in Table 4. Note that each data set consists of SAA passes for which the ISS is either ascending (headed northward) or descending. Consistent differences for these two modes are observed, and result from the directional environment as well as location and directional dependent shield distributions. Such differences in dosimetry of the ascending and descending trajectories has been previously investigated by Dachev et al. (2006) and Hugger et al. (2003).

\subsection{Description of Model Calculations}

The positional data for each mission segment (time, latitude, longitude, altitude, heading plus yaw, pitch, roll) were used as input to the GEORAD environment code to calculate the fluence energy spectra for the GCR and trapped protons. For the calculations pertaining to this work, the cumulative directional fluence along 970 directions has been evaluated in the vehicle coordinate system in the $+X V V$ orientation: (x-axis along velocity vector, z-axis toward earth center, y-axis completing the right-hand system). Yaw, pitch and roll angles are assumed to be zero (neglects the small limit cycle motion), with heading direction specified as angle between direction of flight and geographic north (heading). Directional properties of the SAA protons have been accounted for throughout, and orbit-averaged directional transmission factors for GCR have been used. With respect to solar cycle conditions, the relevant data periods correspond very nearly to solar maximum conditions when GCR and trapped proton fluences are lowest.

Results of fluence calculations integrated over all angles are presented in Figs. 11 and 12 for the trapped protons and GCR, respectively. The proton spectra have been plotted as a total fluence for each mission segment, and reflect the durations thereof. The GCR spectra are plotted in terms of a daily flux for several important GCR ions, and they illustrate the effect of flux reduction at lower energies due to geomagnetic cutoff. The flux spectra 
are then used as input for the 2005 HZETRN transport code to generate dose vs. depth functions (see Figs. 13 and 14).

Table 4. Summary of Results from Data Set Measurements

\begin{tabular}{|c|c|c|c|c|c|c|c|}
\hline \multirow{2}{*}{\multicolumn{2}{|c|}{$\begin{array}{c}\text { Data Set } 1 \\
\text { MDU\#2 (6-9 July) }\end{array}$}} & \multicolumn{2}{|c|}{ Data Set 2} & \multicolumn{2}{|c|}{ Data Set 3} & \multicolumn{2}{|c|}{ Data Set 4} \\
\hline & & \multicolumn{2}{|c|}{ MDU\#1 (6-13 July) } & \multicolumn{2}{|c|}{ MDU\#3 (6-13 July) } & \multicolumn{2}{|c|}{ MDU\#1 (26-28 June) } \\
\hline Asc. & Desc. & Asc. & Desc. & Asc. & Desc. & Asc. & Desc. \\
\hline 4.5 & 2.37 & 6.21 & 4.86 & 4.64 & 4.48 & 2.75 & 9.98 \\
\hline 15.98 & 8.98 & 16.56 & 11.52 & 15.98 & 9.88 & 15.22 & 1.08 \\
\hline 0.66 & 2.04 & 0.91 & 2.24 & 0.96 & 2.18 & 1.97 & 3.67 \\
\hline 1.35 & 6.71 & 1.96 & 2.38 & 1.28 & 1.79 & 0.84 & 10.09 \\
\hline 14.65 & 5.72 & 15.15 & 9.27 & 14.2 & 8.94 & 11.43 & 3.32 \\
\hline 5.73 & 3.24 & 5.74 & 6.59 & 6.31 & 5.56 & 8.49 & 1.63 \\
\hline 7.98 & 8.15 & 0.5 & 1.35 & 8.31 & 5.94 & & 8.06 \\
\hline 14.05 & 0.93 & 9.91 & 10.25 & 14.41 & 8.46 & & 7.75 \\
\hline 2.85 & & 15.4 & 0.96 & 2.67 & 0.97 & & \\
\hline \multirow[t]{10}{*}{16.42} & & 3.94 & 1.07 & 16.4 & 6.63 & & \\
\hline & & 17.18 & 6.78 & 4.76 & 6.12 & & \\
\hline & & 6.85 & 7.97 & 15.96 & 4.74 & & \\
\hline & & 17.57 & 5.34 & 0.64 & 9.12 & & \\
\hline & & 0.64 & 10.71 & 1.42 & 1.64 & & \\
\hline & & 2.59 & 1.99 & 14.47 & 1.94 & & \\
\hline & & 17.75 & 2.26 & 5.57 & 8.88 & & \\
\hline & & 5.78 & 1.9 & & 4.83 & & \\
\hline & & & 9.4 & & & & \\
\hline & & & 6.02 & & & & \\
\hline И 84.17 & 38.14 & 144.64 & 102.86 & 127.9 & 392.1 & 40.7 & 45.58 \\
\hline \multirow[t]{5}{*}{ l Sum } & 122.31 & & 247.5 & & 220.08 & & 86.28 \\
\hline & \multicolumn{7}{|c|}{ Total Mission Dose: } \\
\hline & 444.13 & & 693.2 & & 709.14 & & 284.41 \\
\hline & \multicolumn{7}{|c|}{ Total GCR Dose: } \\
\hline & 321.82 & & 445.7 & & 489.06 & & 198.13 \\
\hline
\end{tabular}

It is assumed that the ISS structure is primarily aluminum alloy ( $\mathrm{Al} 2219)$, and particle flux transport is calculated for traverse through equivalent $\mathrm{g} / \mathrm{cm}^{2} \mathrm{Al}$ amounts. The resultant dose rates are computed for energy deposition in silicon (detector material) and shown in Figs. 13 and 14. It is seen that dose rates for the mission segments are very nearly the same for given aluminum thickness values for GCR with a small separation for trapped protons dependent on specific SAA crossings. For reference, the predicted free space solar maximum GCR dose rates are shown in Fig. 14 to display the effects of geomagnetic transmission factors.

In order to calculate exposure at the specified target locations, directional distributions of shield amounts including the MDU materials are required. A spherical coordinate grid of 970 rays subtending equal solid angles has been implemented. The cumulative angle averaged shield thickness distributions based on the CAD-modeled ISS 6A configuration are shown in Fig. 15. Data measurements for the MDU\#4 location were not available as of this writing, and the MDU\#4 thickness distribution was not used in this study. When the dose vs. depth functions are used in conjunction with the directional thickness distributions, the total mission dose may be predicted. The results of these end-point calculations are given in Table 5; direct comparison with measurements is made in the following section. 


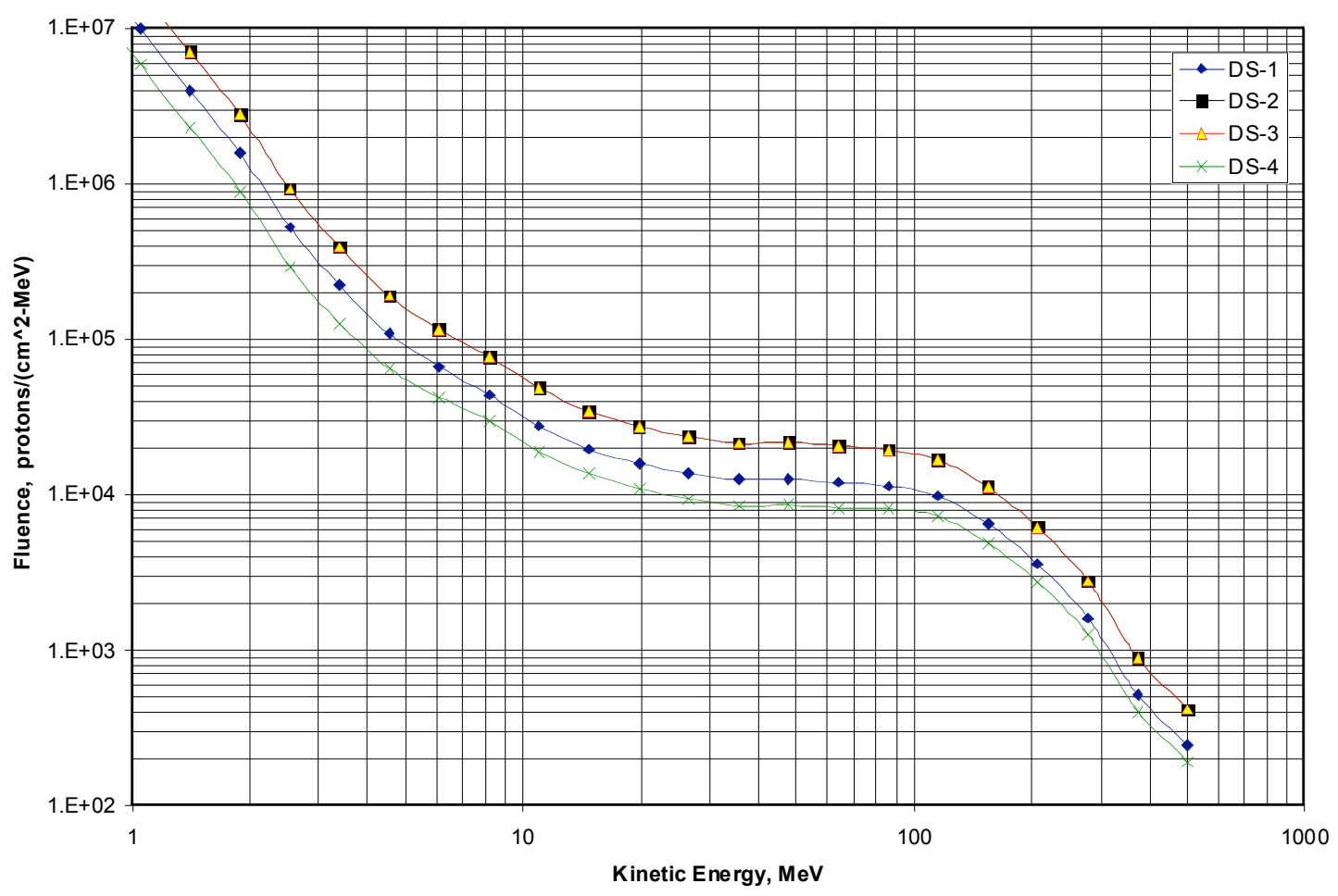

Fig. 11. Calculated trapped proton fluence for data set simulation.

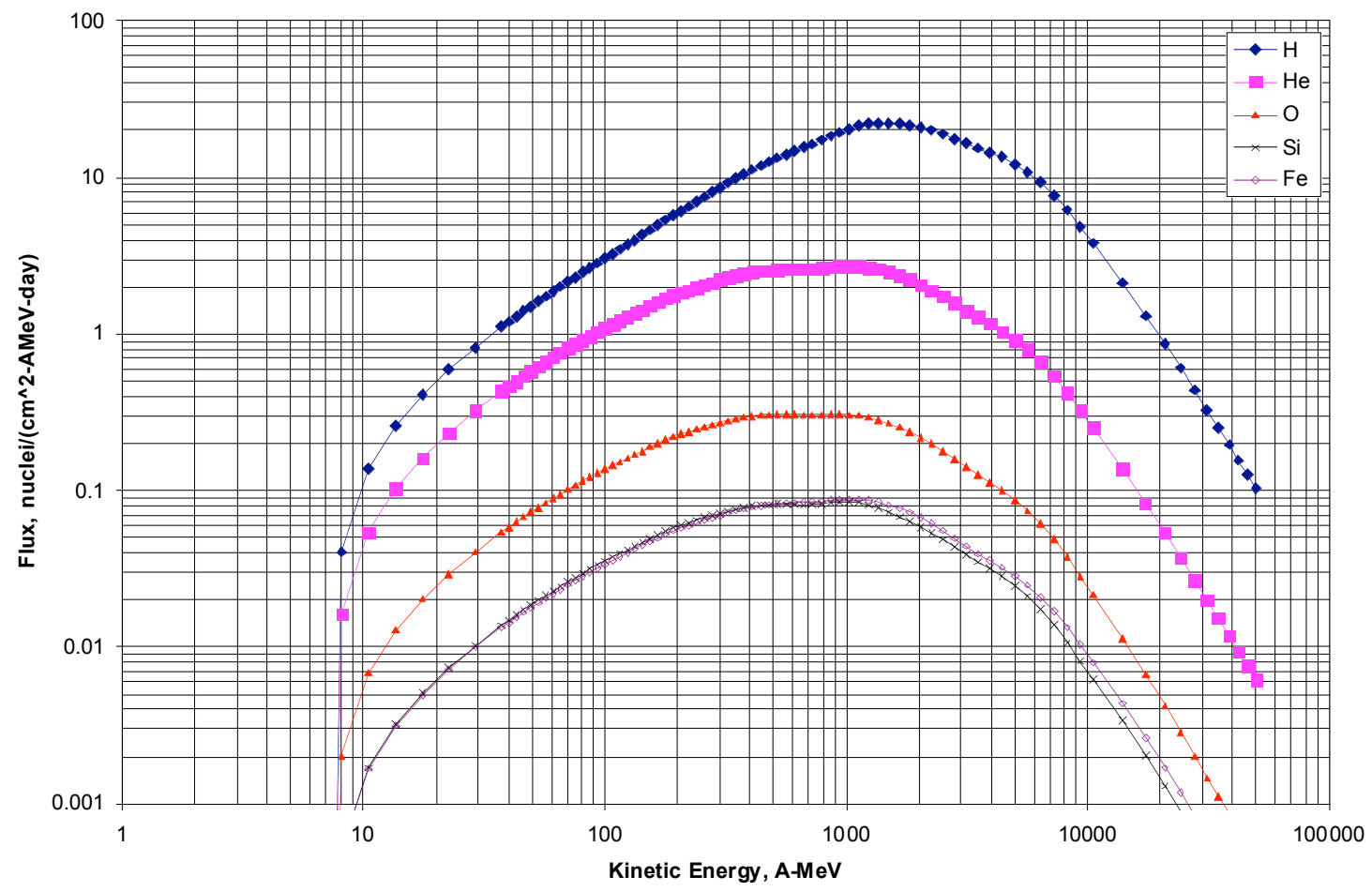

Fig. 12. Calculated daily GCR flux for data period simulation. 


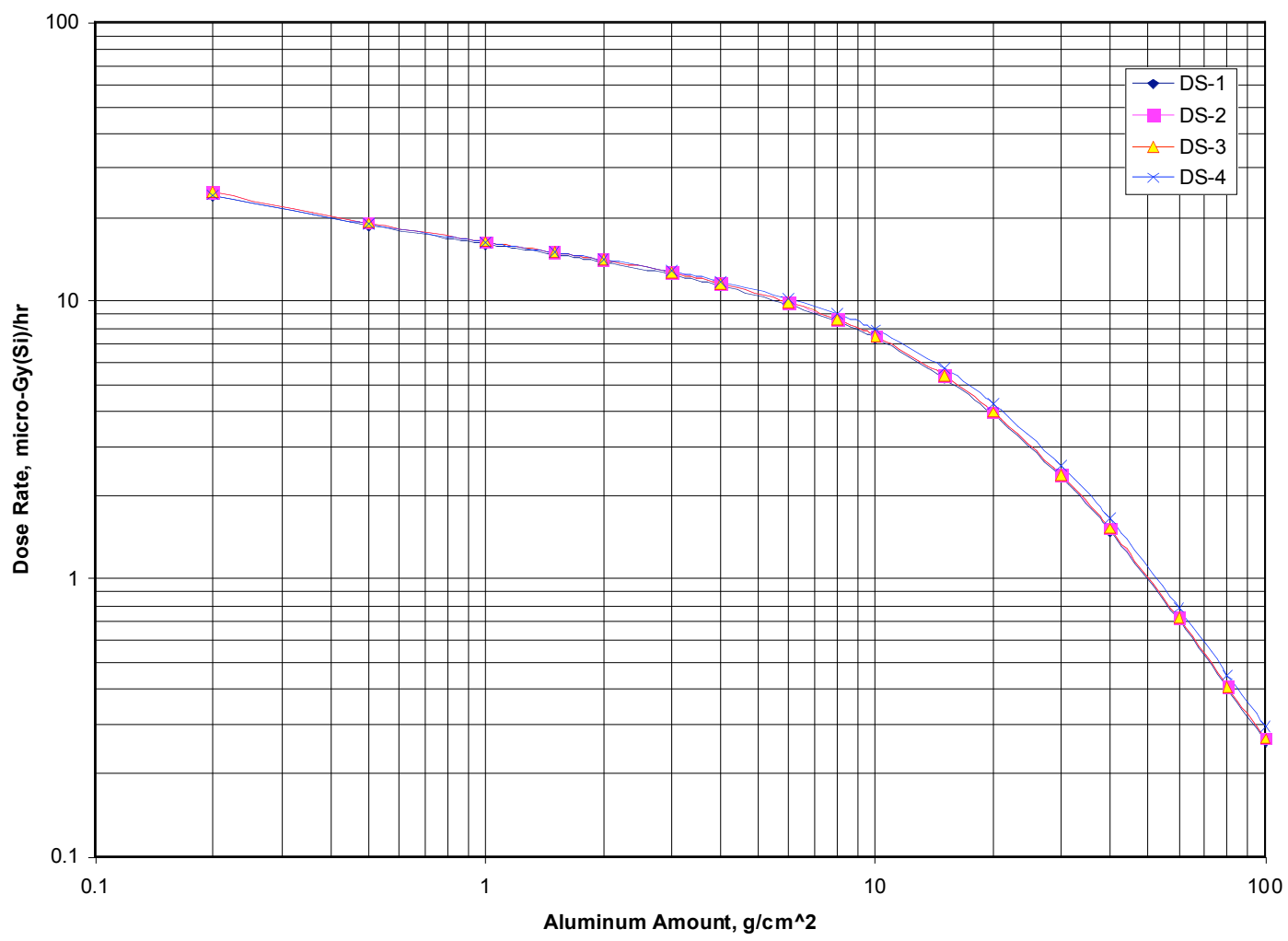

Fig. 13. Transport calculation results (Dose Rate vs. Depth) for simulated data set trapped protons.

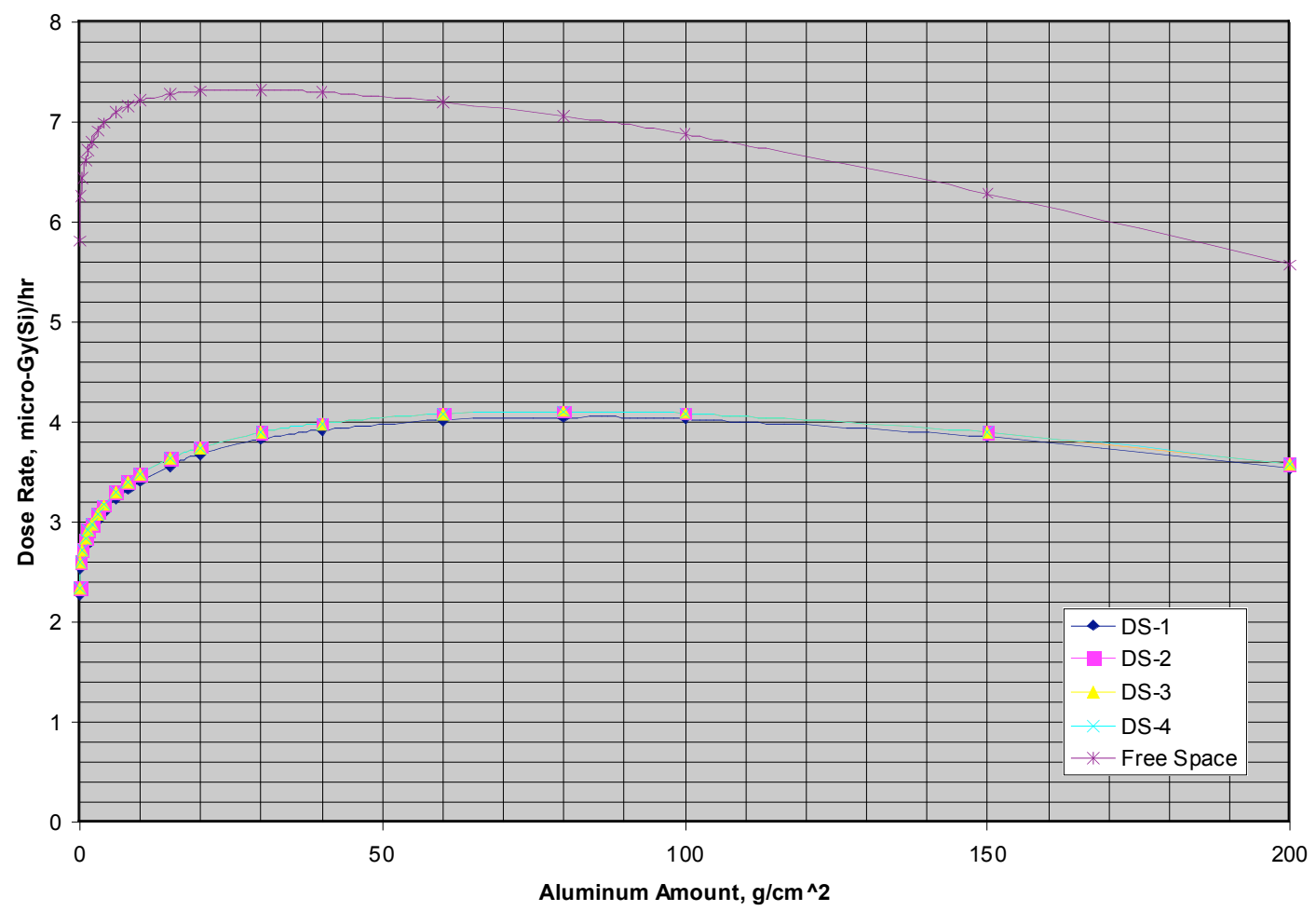

Fig. 14. GCR transport calculations for simulated data sets. 


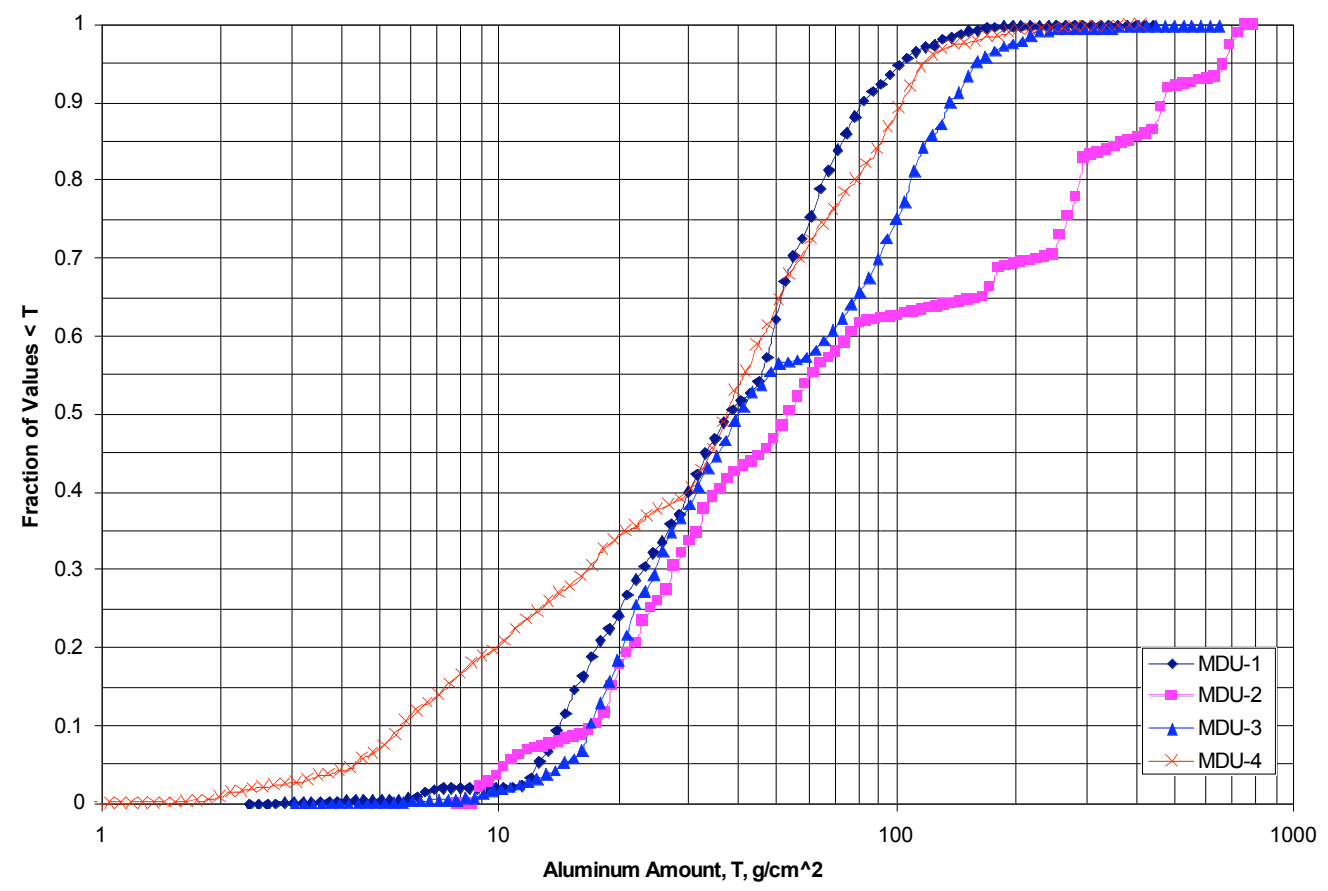

Fig. 15. Cumulative angle averaged distributions of shield amounts for the detector locations.

Another by-product of the 2005 HZETRN code is the calculation of linear energy transfer (LET) at the detector location. A commonly used representation of energy deposition per unit thickness for a given exposure condition is the integral LET spectrum that indicates particle flux resulting in a given energy deposited along the path through the detector. The combined (GCR + trapped protons) LET spectra for the four data sets are shown in Fig. 16. Although the DOSMAP data includes information sufficient to infer LET spectra, at the time of this writing the measured data were not readily available and remain a subject for future analysis.

\begin{tabular}{|c|c|c|c|}
\hline $\begin{array}{c}\text { Data } \\
\text { Set }\end{array}$ & $\begin{array}{l}\text { Trapped } \\
\text { Protons }\end{array}$ & GCR & Total \\
\hline 1 & 193 & 206 & 399 \\
\hline 2 & 240 & 368 & 608 \\
\hline 3 & 104 & 368 & 472 \\
\hline 4 & 111 & 152 & 263 \\
\hline
\end{tabular}

\section{Comparison of Results and Discussion}

A summary chart of the direct comparison of exposure data measured with that calculated is given in Fig. 17 in terms of the average hourly dose rate for each dataset. In general, agreement is fair to good, but despite all efforts to replicate the experiment, differences occur that suggest room for improvement. Since the measured data are of high quality and consistency, a first reaction is to examine possible weaknesses and uncertainties in the theoretical calculations. The trapped environment models used exhibit strong sensitivity to magnetic field specification and altitude (especially in LEO). The GCR component is a rather strong function of assumed solar cycle modulation between maximum and minimum values (in essence, also driven by geomagnetic field transmission factor). The comparison calculations are also strongly influenced by the modeled thickness distributions around the target points (detector locations), particularly with respect to the trapped proton exposures and further refinement of the ISS model may reduce differences. The distribution of shield thickness is also inherently coupled to the modeled directionality of the trapped protons in the SAA. The solar cycle modulation, shield amount distributions, and trapped proton directional flux have all been addressed in the present calculations with the best information available. 


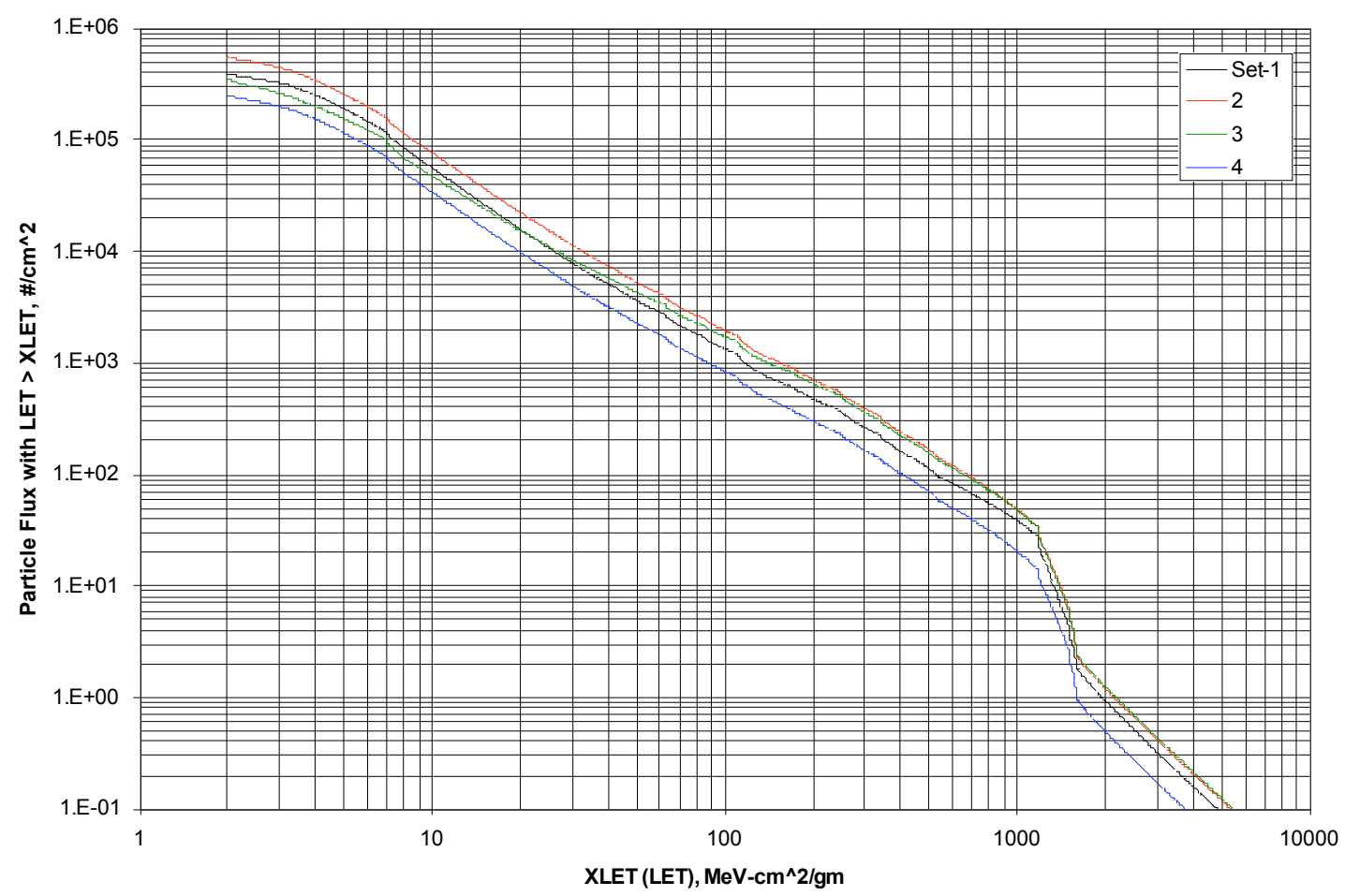

Fig. 16. Particle flux LET spectra calculated for simulated data set environments and detector locations,

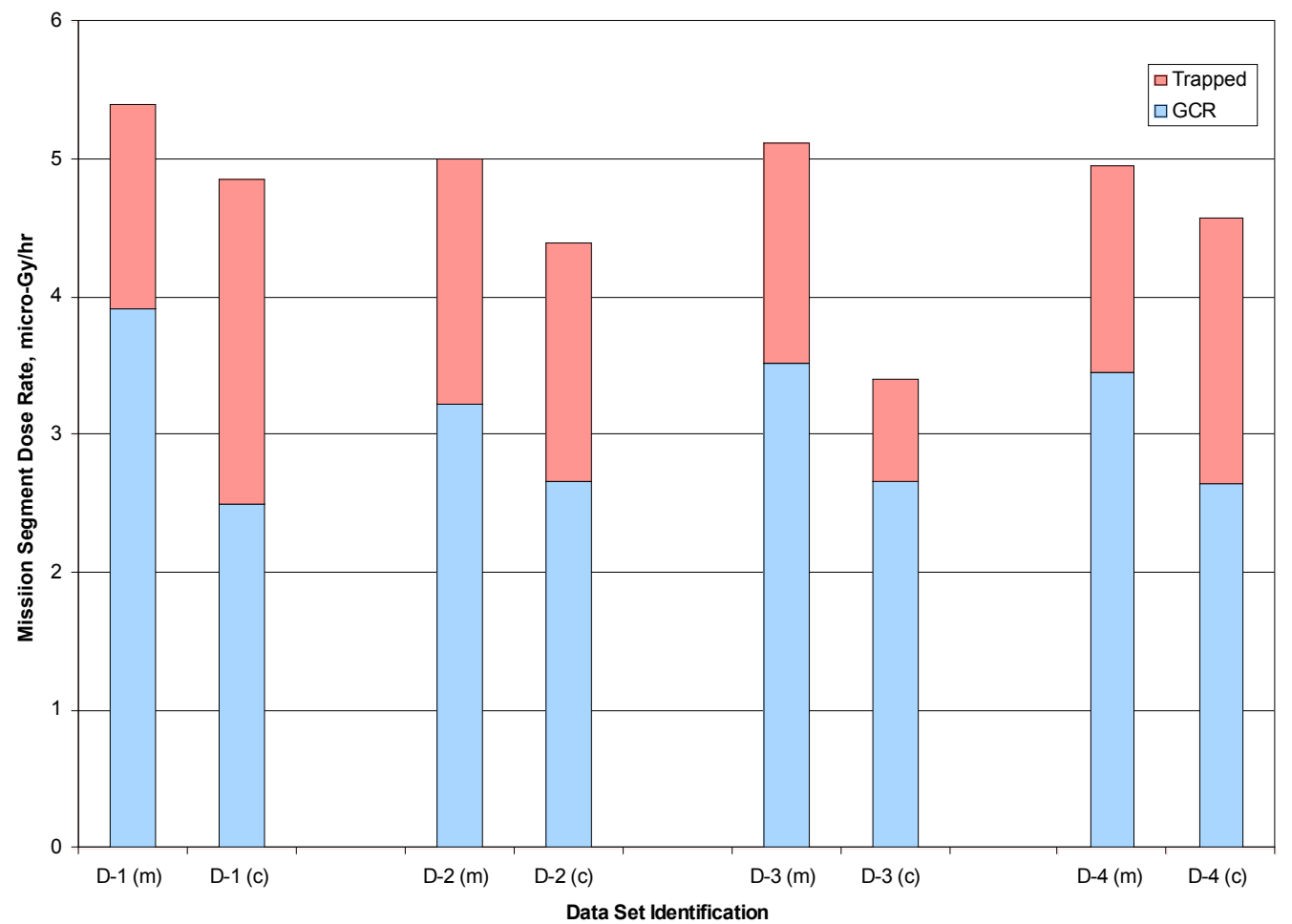

Fig. 17. Comparison of measured (m) with calculated (c) average dose rates. 
Another factor that could impact the calculations is that of the detectors' directional response (field of view) which has been addressed in the present work as the asymmetrical distribution of the instrument mass but not with respect to the directional response of the silicon detector (see Fig. 7). This effect could be of significant importance for the less penetrating trapped proton component. These considerations suggest areas for examination in future analysis. It does appear that the GCR contributions of the four datasets as predicted by the models in Fig. 17 are consistently low by about 15-20 percent and may be due to use of the older implementation BadhwarO'Neill (1995) GCR model wherein the decades older data of the model are extrapolated using only current and historic Sunspot Numbers (Wilson et al. 2002, Kim et al. 2006), and the use of the new Badhwar-O'Neill GCR model (O’Neill 2006) is expected to improve the modeled result and reduce these differences.

\section{Concluding Remarks}

Consistent differences in the GCR comparison exist in that calculations result in lower predicted exposures for all mission segments. These exposures are sensitive to the strength of the solar maximum condition. A weaker solar maximum than that used in the present calculations would increase these predicted exposures. For the trapped exposures, differences are not consistent; very good agreement is indicated for data sets 2 and 4, whereas significant differences are seen for sets 1 and 3. For set 1, greater trapped exposure is predicted while for set 3 the trapped exposure is substantially underestimated. Since the trapped proton spectra are relatively "soft", they exhibit high sensitivity to effective shield amounts. Additionally, the trapped environment is modeled with solar cycle modulation and imposed drift of the aging AP8MIN and AP8MAX static models. Future analysis of data such as that provided by the LIULIN system could well lead to up-grading the trapped environment with possible definition and inclusion of inherent short-term variability of flux intensities.

\section{References}

Alsmiller, R.G., High-energy nucleon transport and space vehicle shielding. Nucl. Sci. \& Eng. 27: 158-189; 1967.

Alsmiller, R.G., Irving, D.C., Moran, H.S., Validity of the straightahead approximation in space-vehicle shielding studies. Nucl. Sci. \& Eng. 32: 56-61; 1968.

Atwell, W. Beever, E.R., Hardy, A.C., Richmond, R.G., Konradi, A., Space radiation shielding analysis and dosimetry for the Space Shuttle Program. In High-Energy Radiation Background in Space, Eds. A. C. Rester \& J. I. Trombka, AIP Conference Preceedings 186, New York, 289-296; 1989.

Badhwar, G.D., O’Neill, P.M., An improved model of galactic cosmic radiation for space exploration missions. Nucl. Tracks Radiat. Meas. 20: 427-410; 1995.

Badhwar, G.D., Patel, J.D., Atwell, W., Cucinotta, F.A., Wilson, J.W., In-flight radiation measurements on STS-60. Radiat. Meas. 26:17-34; 1996.

Badhwar, G.D., Radiation dose rates in Space Shuttle as a function of atmospheric density. Radiat. Meas. 30:401414; 1999.

Benton, E.R., Radiation dosimetry at aviation altitudes and in low Earth orbit. $\mathrm{PhD}$ dissertation, University College, Belfield, Dublin, May 2004.

Bertini, H.W., Guthrie, M.P., Culkowski, A.H. Nonelastic Interactions of Nucleons and $\pi$-Mesons with Complex Nuclei at Energies Below 3 GeV. ORNL TM-3148, 1972.

Blanchard, R.C., Hess, W.N., Solar cycle changes in inner zone protons. J. Geophys. Res. 69:3927; 1964.

Cain, J.C. Hendricks, S.J., Langel, R.A., Hudson, A proposed model for the international geomagnetic reference field-1965. J. Geomag. Geoelec. 19: 335-355; 1967.

Cucinotta, F. A., Calculations of cosmic ray helium transport in shielding materials. NASA TP-3354, 1993.

Curtis, S.B., Wilkinson, M.C., The Heavy Particle Hazard-What Physical Data Are Needed? Proceedings of the National Symposium on Natural and Manmade Radiation in Space, E.A. Warman, ed., NASA TM-X2440, 1007-1015; 1972.

Dachev, T., Atwell, W., Semones, E., Tomov, B., Reddel, B., Observations of SAA distributions by Liulin E-094 instrument on ISS. Adv. Space Res., 37: 1672-1677; 2006.

De Angelis, G., J.M. Clem, P. Goldhagen, J.W. Wilson, A new dynamical Atmospheric Ionizing Radiation (AIR) model for epidemiological studies, Adv. Space Res., 32(1), 17-26, 2003.

Heckman, H.H., Nakano, G.H., East-west asymmetry in the flux of mirroring geomagnetic trapped protons. $J$. Geophys. Res. 68:2117-2120; 1963.

Heynderickx, D., Comparison between methods to compensate for the secular motion of the South Atlantic Anomaly. Radiat. Meas. 26: 369-373; 1996. 
Hugger, C.P., Nealy, J.E., Clowdsley, M.S., Wilson, J.W., Qualls, G.D., Atwell, W., Cucinotta, F.A., Golightly, M.J., Semones, E., Shavers, M.R., Preliminary evaluation of an ISS radiation shielding model. AIAA 20036620, 2003.

Huston, S.L. Pfitzer, K.A., Space environment effects: Low-altitude trapped radiation model. NASA/CR-1998208593, 1998.

Jenkins, R. W.; Ifedili, S. O.; Lockwood, J. A.; Razdan, H., The energy dependence of the cosmic-ray neutron leakage flux in the range 0.01-10 MeV.J. Geophys. Res. 76:7470-7478; 1971.

Jensen, D.C., Cain, J.C. An interim geomagnetic field. J. Geophys. Res. 67: 3568-3569; 1962.

Kern, J.W., A note on vector flux models for radiation dose calculations. Radiat. Meas 23: 43-48; 1994.

Kim, M.H., Wilson, J.W., Cucinotta, F.A., A solar cycle statistical model for the projection of space radiation environment. Adv. Space Res. 37: 1741-1748; 2006.

Korff, S. A., Mendell, R. B., Merker, M., Light, E. S., Verschell, H. J., and Sandie, W. S., Atmospheric Neutrons. NASA CR 3126, 1979.

Lockwood, J. A., Neutron measurements in space. Space Sc. Rev. 14: 663-719; 1972.

McCormack, P.D., Radiation dose and shielding for the Space Station. Acta Astronaut. 17: 231-241; 1988.

O'Neill, P.M., Badhwar-O'Neill galactic cosmic ray model update based on advanced composition explorer (ACE) energy spectra from 1997 to present. Adv, Space Res., 37(9): 1727-1733; 2006.

Pfitzer, K.A., Radiation dose to man and hardware as a function of atmospheric density on the $28.5^{\circ}$ space station orbit. McDonald Douglas Space Systems Co. Report No. H5387A, Huntington Beach, CA, 1990.

Preszler, A.M., Simnett, G.M., White, R.S., Earth albedo neutrons from 10-100 MeV. Phys. Rev. Letters 28:982984; 1972.

Qualls, G.D., Wilson, J.W., Cucinotta, F.A., Nealy, J.E., Hugger, C.P., Atwell, W., Shavers, M.R., International Space Station Radiation Shield Augmentation Optimization. Space 2003 Conference. AIAA 2003-6222, 2003.

Quenby, J.J., Webber, W.R., Cosmic-ray cutoff rigidities and the Earth's magnetic field, Phil. Mag. 4: 90; 1959.

Ranft, J., The FLUKA and KASPRO hadronic cascade codes. Computer Techniques in Radiation Transport and Dosimetry, W. R. Nelson and T.M. Jenkins, eds, Plenum Press, pp. 339-371, 1980.

Sawyer, D.M., Vette, J.I., AP-8 Trapped Proton Environments for Solar Maximum and Solar Minimum. NSSDC/WDC-A-R\&S 76-06, 1976.

Shavers, M. R., Frankel, K., Miller, J., Schimmerling, W., Townsend, L. W., Wilson, J. W., The fragmentation of $670 \mathrm{~A} \mathrm{MeV}$ neon-20 as a function of depth in water. III. Analytic multigeneration transport theory. Radiat. Res., 134(1): 1-14; 1993.

Shinn, Judy L., John, Sarah, Tripathi, Ram K., Wilson, John W., Townsend, Lawrence W., and Norbury, John W., Fully-Energy Dependent HZETRN (A Galactic Cosmic-Ray Transport Code). NASA TP-3243, November 1992.

Smart, D.F., Shea, M.A., Geomagnetic transmission functions for $400 \mathrm{~km}$ altitude satellite. $18^{\text {th }}$ International Cosmic Ray Conference-Conference Papers MG Sessions, Vol. 3, Tata Inst. of Fundamental Research (Colaba, Bombay) pp. 419-422; 1983.

Tai, H., Bichsel, H., Wilson, J. W., Shinn, J. L., Cucinotta, F. A., Badavi, F. F., Comparison of Stopping Power and Range Data Bases for Radiation Transport Study. NASA TP-3644, 1997.

Tweed, J., Walker, S.A., Wilson, J.W., Cucinotta, F.A., Tripathi, R.K., Blattnig, S., Mertens, C.J., Computational methods for the HZETRN code. Adv. Space Res. 35: 194-201; 2005.

Uchihori, Y., Kitamura, H., Fujitaka, K., Dachev, T.P., Tomov, B.T., Dimitrov, P.G., Matviichuk, Y., Analysis of the calibration results obtained with Liulin- $4 \mathrm{~J}$ spectrometer-dosimeter on protons and heavy ions. Rad. Meas. 35: $127-134 ; 2002$.

Vette, J.I., The NASA/National Space Science Data Center Trapped Radiation Environmental Model Program (1964-1991). NSSDC/WDC-A-R\&91-29. 1991.

Wilson, J.W., Khandelwal, G.S., Proton dose approximation in convex geometry. Nucl. Tech. 23: 298-305; 1974.

Wilson, J.W., Lamkin, S.L., Perturbation theory for charged-particle transport in one dimension. Nuclear Sci \& Eng. 57:292-299; 1975.

Wilson, J.W., Analysis of the theory of high-energy ion transport. NASA TN D-8381, 1977.

Wilson, J.W., Lamkin, S.L., Farhat, H., Ganapol, Townsend, L.W., A Hierarchy of Transport Approximations for High Energy Heavy Ion (HZE) Transport. NASA TM-4118, 1989.

Wilson, J.W., Townsend, L.W., Schimmerling, W., Khandelwal, G.S., Khan, F., Nealy, J.E., Cucinotta, F.A., Simonsen; L.C., Shinn, J.L., Norbury, J.W., Transport Methods and Interactions for Space Radiations. NASA RP-1257, 1991.

Wilson, J.W., Kim, M.-H. Y., Shinn, J.L., Tai, H., Cucinotta, F.A., Badhwar, G.D., Badavi, F.F., Atwell, W., Solar Cycle Variation and Application to the Space Radiation Environment. NASA TP 1999-209369, 1999.

Wilson, J.W., Overview of radiation environments and human exposures. Health Physics 79:470-494; 2000. 
Wilson, J.W., Tweed, J., Tai, H., and Tripathi, R.K., A simple model for straggling evaluation. Nucl Inst \& Methods B 194: 389-392; 2002a.

Wilson, J.W. Badavi, F.F., Kim, M.H., Clowdsley, M.S., Heinbockel, J.H., Cucinotta, F.A., Badhwar, G.D., Atwell, W., Huston, S.L., Natural and induced environment in low Earth orbit. NASA/TM-2002-211668, $2002 \mathrm{~b}$.

Wilson, J.W., Korte, J.J., Sobieski, J., Badavi, F.F., Chokshi, S.M., Martinovic, Z.N., Cerro, J., Qualls, G.D., Radiation shielding, MDO processes, and Single Stage to Orbit design. Space 2003 conference, AIAA 2003$6259,2003$.

Wilson, J.W., Cucinotta, F.A., Schimmerling, W., Emerging radiation health-risk mitigation technologies. Space Technology Applications \& Information Forum, STAIF-2004, AIP Conference Publications, 2004.

Wilson, J.W., Tripathi, R.K., Mertens, C.J., Blattnig, S.R., Clowdsley, M.S., Cucinotta, F.A., Tweed, J., Heinbockel, J.H., Walker, S.A., Nealy, J.E., Verification and Validation: High Charge and Energy (HZE) Transport Codes and Future Development. NASA/TP-213784, 2005.

Wilson, J.W., Tripathi, R.K., Badavi, F.F., Cucinotta, F.A., Standardized Radiation Shield Design Method: 2005 HZETRN. 2006 International Conference on Environmental Systems, SAE Paper 2006-01-2109, $2006 \mathrm{a}$.

Wilson, J.W., Cucinotta, F.A., Golightly, M.J., Nealy, J.E., Qualls, G.D., Badavi, F.F., De Angelis, G. Anderson, B.M., Clowdsley, M.S., Luetke, N., Zapp, N., Shavers, M.R., Semones, E., Hunter, A., International space station: A testbed for experimental and computational dosimetry. Adv. Space Res. 37: 1656-1663; $2006 \mathrm{~b}$.

Wilson, J.W., Nealy, J.E., Dachev, Ts., Tomov, B.T., Cucinotta, F.A., Badavi, F.F., De Angelis, G., Leutke, N., Time serial analysis of the induced LEO environment within the ISS 6A. Adv. Space Res., under review, 2007. 